\title{
Lugalbandas Königtum und das Feuer des Prometheus
}

\author{
Merkmale, Funktionen und Interpretationen von Mythen über \\ eine Herkunft vom Himmel
}

\author{
für Claus Wilcke \\ den großartigen Lehrer, Mentor \\ und langjährigen Wegbegleiter \\ in herzlicher Verbundenheit
}

\begin{abstract}
Mythical narratives (Erzählstoffe) about someone or something coming down from heaven have a strong claim to relevance in the interpretation of, and in coping with, the human condition. The attribution of a heavenly origin to certain beings, phenomena, or objects, marks them out as special because they are either viewed (a) as prototypical or (b) as divine or numinous. Using the example of the myth of ZEUS BRINGING THE PALLADION DOWN TO EARTH, we will show the extent to which (a) can affect the interpretation of myths where the prototypical character is not explicitly mentioned. The consequences for the interpretation of myths which can result from (b) are illustrated by the myths of THE GODS MAKING LUGALBANDA A DIVINE RULER while the goddess Innana is taking away the kingship from the former ruler Enmerkara, and PROMETHEUS STEALING THE FIRE. Because of its divine origin, the taming of fire is seen in these mythical traditions not as a cultural but as a cult technique, related not primarily to the culinary arts but rather to cultic practices.
\end{abstract}

\section{Mythen über eine Herkunft aus dem Himmel: Merkmale}

In mythischen Erzählstoffen aus antiken Kulturen wird immer wieder davon erzählt, dass etwas „aus dem Himmel kommt“. Allerdings ist diese Wendung, dass etwas „aus dem Himmel kommt“, eine verkürzende und abstrahierende Redeweise. In ausformulierten Mythen erscheint ein solcher Transfer aus dem Himmel in konkreter, genauer in personalisierter Form. Das bedeutet, dass ein (in aller 
Regel: göttlicher) Handlungsträger für einen solchen Sphärenwechsel verantwortlich ist: Entweder kommt jemand vom Himmel, oder jemand veranlasst, dass etwas aus dem Himmel kommt.

In den meisten Fällen handeln solche mythischen Stoffe davon, dass etwas oder jemand vom Himmel auf die Erde kommt, auch wenn dies nicht immer explizit gemacht wird; in selteneren Fällen kann der Zielpunkt auch in anderen Bereichen wie bspw. in der Unterwelt oder im Meer liegen. In einem Fall entsteht durch das Herabkommen eines Hauses vom Himmel sogar erst die Erde ${ }^{1}$.

Wenn in Konkretionen mythischer Stoffvarianten thematisiert wird, dass etwas oder jemand vom Himmel in einen anderen Bereich gelangt, dann können solche Hinweise auf den konkreten, einzelnen Vorgang eines solchen Sphärenwechsels abzielen. Ein Beispiel dafür ist das konkrete mythische Hylem „Innana bringt das Himmelshaus für die Erde“, das sich abstrahieren und damit verallgemeinern lässt zum Hylemschema „Gottheit NN bringt einen Tempel für die Erde“. Manchmal kann ein solches Hylem aber auch stellvertretend oder nach Art einer Überschrift für den ganzen Mythos stehen, in welchem dieses eine Hylem einen zentralen Handlungsbestandteil darstellt; in einem solchen Fall ist „Innana bringt das Himmelshaus für die Erde“ als Hyperhylem anzusehen und „Gottheit NN bringt einen Tempel für die Erde“ als ein Hyperhylemschema ${ }^{2}$.

Die einzelnen Studien dieses Bandes gehen der Frage nach, welche Themenkomplexe oder auch Problembereiche in den untersuchten Quellen narrativ so verarbeitet werden, dass sie als Ergebnis eines Sphärenwechsels gedeutet werden, der seinen Ausgangspunkt im Himmel nimmt. Im Ergebnis lässt sich festhalten, dass der genannte Transfer mit verschiedenen, aber nicht mit unüberschaubar vielen unterschiedlichen Themen und Problemen in Zusammenhang gebracht werden kann. So sind es beispielsweise die Themenkomplexe „Gerechtigkeit und Ordnung “, die in mythischen Stoffen unter anderem mit dem Vorgang „X kommt vom Himmel“ in Zusammenhang gebracht werden können, der in verschiedenen Kulturen und Erzählstoffen unterschiedlich konkretisiert wird.

In Hinblick auf das Thema „Ordnung (des menschlichen Zusammenlebens)“ besteht eine solche Konkretion in Mesopotamien bspw. darin, dass eine Gottheit Insignien des Königtums vom Himmel herabbringt. Dieser Vorgang kann auch abstrakter formuliert werden, bspw. durch die Aussage, dass „das Königtum vom

1 Vgl. Beitrag A. Zgoll, Wie der erste Tempel auf die Erde kommt.

2 S. zu den hier verwendeten Unterscheidungen und der entsprechenden Terminologie den einführenden Beitrag von C. Zgoll, Grundlagen der hylistischen Mythosforschung in diesem Band. Der Einfachheit halber wird im Folgenden nicht mehr eigens erwähnt, dass ein Hylemschema auch als Hyperhylemschema fungieren kann. 
Himmel kommt“; aber auch hinter solchen abstrakteren, abkürzenden Formulierungen steckt in der Regel ein mythischer Erzählstoff, der in auserzählter Form deutlich konkreter, also gegenständlich und v. a. personalisiert vorgestellt wur$\mathrm{de}^{3}$.

In Bezug auf den Themenkomplex „Gerechtigkeit“ kommt bspw. in Ägypten die personifizierte Rechtlichkeit namens Maat vom Himmel ${ }^{4}$. Auch in Griechenland wird das personifizierte Recht als Garant guten menschlichen Zusammenlebens als etwas beschrieben, das aus dem Herrschaftsbereich des Zeus kommt, allerdings zu einem späteren Zeitpunkt in der Geschichte die Erde verlässt und sich wieder in den Himmel (bzw. auf den Olymp) zurückzieht ${ }^{5}$; es handelt sich um Dike, die Tochter von Themis und Zeus ${ }^{6}$.

Verschiedene Vorstellungen oder Wirklichkeitserfahrungen können narrativ gleich verarbeitet und damit mythisch gleich oder doch sehr ähnlich plausibilisiert werden, indem bspw. für verschiedene Entitäten gleichermaßen erzählt wird, dass sie „vom Himmel gekommen sind“; umgekehrt können interessanterweise selbst innerhalb einer Kultur für die Erklärung der Herkunft derselben Entitäten verschiedene mythische Stoffe zum Einsatz kommen. Anders ausgedrückt: Für die Betonung einer göttlichen Herkunft muss nicht immer eine himmlische Herkunft als Grund angegeben werden.

In Mesopotamien ist es u. a. der Abzu, der Süßwasserozean, aus dem heraus vor allem der Gott Enki wirkmächtig agiert, in Griechenland ist der Olymp Wohnund Wirkungsstätte der Götter, der teilweise aber auch mit dem Himmel gleichgesetzt ist ${ }^{7}$. Während der ancile-Schild von Jupiter aus dem Himmel geschickt wird $^{8}$, stellt der Schmiedegott Vulcanus Rüstung und Schild für Aeneas in einer

3 Vgl. für den (altorientalischen) Fall des vom Himmel herabkommenden Königtums C. Zgoll 2019, 197-204; ausführlich den Beitrag von Gabriel in diesem Band.

4 S. den Beitrag von Naether in diesem Band. Vgl. auch JHWH, der im Alten Testament zu Mose auf den Berg Sinai hinabsteigt (vgl. Ex 19,18.20; 34,5), um dem Volk Israel Gesetze zu geben.

5 Erst bei den römischen Dichtern, wo sie Virgo oder Astraea heißt, vgl. Verg. ecl. 4,6; Ov. met. $1,149 \mathrm{f}$.

6 Hes. theog. 902.

$7 \mathrm{Zu}$ den Schwierigkeiten der Differenzierung von Olymp und Himmel sowie deren teils synonyme Verwendung siehe den Beitrag C. Zgoll, Göttergaben und Götterstürze in diesem Band.

8 Vgl. Beitrag Jäger. 
unterirdischen Höhle her ${ }^{9}$, und etliche Mythen aus verschiedenen antiken Kulturen berichten davon, dass Wichtiges aus dem Bereich der Unterwelts-Gottheiten auf die Erde gelangt ${ }^{10}$.

Ein Beispiel aus dem antiken Mesopotamien soll das noch etwas genauer veranschaulichen: Wenn Innana das „Himmelshaus“ und damit den ersten Tempel vom Himmel für die Erde bringt, dann schließt diese offenbar mit Uruk verbundene Tradition nicht aus, dass nach einer anderen Überlieferung der Gott Enki den (implizit ebenfalls gemeint: ersten) Tempel aus dem Abzu, dem unterirdischen Süßwasserozean dort entstehen lässt, wo die Stadt Eridu liegt. Der Tempel kommt hier also nicht aus dem Himmel, sondern aus dem $\mathrm{Abzu}^{11}$. Die mit dieser Eridu-Tradition verbundenen Aussagen sind genauso komplex und verschieden, wie manche Aussagen über den Tempel, der nach der Uruk-Tradition vom Himmel kommt; wie dort der Tempel einerseits vom Himmel kommt, andererseits der große Himmel selbst ist, so berührt nach dem Mythos im Text Enkis Tempelstiftung (traditionell: Enkis Fahrt nach Nippur) der Tempel den Abzu, er grenzt an ihn, er steht in seiner Mitte und wird schließlich selbst mit dem Abzu gleichgesetzt.

Nicht in jeder Tradition kommt also der Tempel vom Himmel, sondern es existieren verschiedene mythische Erzählungen über Tempel-Ursprünge. Bei allen Unterschieden der mythischen Aussagen lässt sich als Gemeinsamkeit erkennen, dass das Verhältnis zwischen Tempel und Herkunftsort in beiden Fällen ähnlich komplex ist, da in beiden Fällen Tempel und Herkunftsort zum Teil gleichgesetzt werden. Noch wichtiger aber ist der Umstand, dass im Ergebnis beide Erzählstoffe

9 Vgl. Beitrag C. Zgoll, Göttergaben und Götterstürze.

10 Andere Beispiele für Mesopotamien, wo Wichtiges oder Positives nicht aus dem Himmel kommt: Schafzucht und Getreideanbau kommen nach dem Streitgespräch Mutterschaf und Getreide vom „Urhügel“ der Götter zu den Menschen (vgl. dazu die neue Übersetzung und Interpretation bei A. Zgoll 2012, 60-62; zu beachten ist, dass dort Z. 26-34 ein Subjektswechsel nicht angezeigt wird); nach Enlil und Ninlil kommen Pflanzenwachstum und Bewässerung aus der Unterwelt (A. Zgoll 2013); in Innana und Enki bringt Innana göttliche Machtmittel (me) aus dem Süßwasserozean Abzu, in Innanas Gang zur Unterwelt bringt Innana solche Machtmittel aus dem Totenreich (A. Zgoll 2020 und 2020a; A. Zgoll/C. Zgoll 2020); nach Ritualtexten kommt Schlechtes wie Krankheit(sdämonen) häufig auch aus der Unterwelt (vgl. z. B. die Ritualtexte gegen Totengeister und Dämonen namens u d u g hu ul / utukku lemnūtu).

11 Die verschiedene Herkunft hängt jeweils mit der besonderen, festen Relation der einzelnen Gottheiten zu bestimmten kosmischen Räumen zusammen (Enki als Gott des Süßwasserozeans bzw. Innana als Himmelsgöttin). 
auf eine ähnliche Funktionalisierung (dazu gleich mehr) hinauslaufen, nämlich den Tempel als etwas zu kennzeichnen, das numinosen Ursprungs ist ${ }^{12}$.

\section{Mythen über eine Herkunft aus dem Himmel: Funktionen}

\subsection{Funktionsbegriff und Grundfunktion mythischer Stoffe}

Wenn es um die Frage nach Funktionen von Mythen geht, ist es aufgrund der Unschärfe des Funktionsbegriffs sinnvoll, terminologisch zu differenzieren zwischen Grundfunktionen von Mythen (was jeder Mythos leistet), primären und sekundären Funktionalisierungen (wozu im Einzelnen eine mythische Stoffvariante eingesetzt wird) und Metafunktionen (welche Auswirkungen der Einsatz einer mythischen Stoffvariante auf diejenigen hat, die sie einsetzen) ${ }^{13}$.

Primäre Funktionalisierungen zielen auf den Einsatz von spezifischen, sekundäre auf den Einsatz von unspezifischen Leistungen, die Mythenvarianten erbringen (können); das eine meint also einen Einsatz von Mythenvarianten zu Zwecken, zu denen sie gedacht bzw. gemacht sind (z. B. etwas zu erklären oder zu legitimieren), das zweite eine Verwendung von Mythen zu Zwecken, zu denen sie eigentlich nicht gedacht sind (z.B. einen philosophischen Gedankengang zu illustrieren oder einer Liebeswerbung mehr Nachdruck zu verleihen).

Die Grundfunktion mythischer Stoffe besteht darin, dass sie als äußerst flexible und komplexe Instrumente dazu dienen können, transzendierende Auseinandersetzungen mit Erfahrungsgegenständen überhaupt zum Ausdruck zu bringen und kommunizierbar zu machen. Da diese Grundfunktion allen Mythen gemein ist, soll gleich auf die verschiedenen Möglichkeiten der Funktionalisierungen speziell von mythischen Hylemen bzw. Hylemsequenzen näher eingegangen werden, die ein Herabkommen aus dem Himmel thematisieren.

12 Zum hier und im Folgenden verwendeten Begriff „numinos“ s. die Ausführungen in Abschnitt 3.2.

13 Zu dieser Differenzierung des Funktionsbegriffs s. im Einzelnen C. Zgoll 2019, 418-428. 


\subsection{Funktionalisierungen: Anzeiger für Bedeutsamkeit und Legitimation}

Primäre Funktionalisierungsmöglichkeiten hinsichtlich der oben genannten Beispiele von Königtum und Maat bestehen darin zu zeigen, von wem veranlasst, unter welchen Umständen und dass überhaupt Ordnung und Gerechtigkeit auf Erden etabliert worden sind und, damit zusammenhängend, inwiefern sie dadurch legitimiert sind und als wichtig oder sogar unantastbar zu gelten haben. Die transferierten Objekte besitzen in der Welterfahrung ihrer Kulturen Wichtigkeit; dies zeigt sich unter anderem an der Bedeutsamkeit der mit dem Sphärenwechsel verbundenen Objekte selbst $\mathrm{t}^{14}$, aber auch an der Bedeutsamkeit der damit verbundenen handelnden Subjekte ${ }^{15}$ und darüber hinaus an den Empfängern oder Orten, zu denen die transferierten Objekte gelangen ${ }^{16}$ :

- Subjekte: Es handelt sich bei den Subjekten oder Veranlassern von Sphärenwechseln in der Regel um hochgestellte Gottheiten.

- Objekte: Das, was aus dem Himmel kommt, ist in der Regel bedeutsam, entweder (in vielen Fällen) etwas außerordentlich Gutes wie Tempel, Götterstatuen oder königliche Insignien (z.B. ein Zepter), oder (seltener) etwas Bedrohliches wie bspw. von hohen Göttern geschickte Flutkatastrophen oder andere numinose Wesen (z. B. Dämonen), die Strafe oder Leid bringen können. Ob etwas wie Bestrafung als negativ einzustufen ist, hängt allerdings sehr von der jeweiligen Perspektive ab. Aus der Sicht eines Übeltäters ist die drohende Strafe tatsächlich etwas Bedrohliches; aus der Perspektive der Menschen, die durch ihn zu Schaden gekommen sind, oder der Götter, die durch ihn bspw. geschmäht wurden, sind Strafen das Sinnbild für Ordnung und Rechtlichkeit ${ }^{17}$.

- Empfänger und Orte: Gerade gute Gaben von oben gelangen zu herausragenden menschlichen Empfängern oder besonderen irdischen Orten wie bspw. das königliche Zepter zum König Šulgi von Ur, der numinose Schild

14 Vgl. Auswertungsbeitrag A. Zgoll, Wer oder was vom Himmel kommt.

15 Vgl. Auswertungsbeitrag Cöster-Gilbert, Akteure von Transfers vom Himmel.

16 Vgl. Auswertungsbeitrag Kärger, Ausgangs- und Zielpunkte des Transfers vom Himmel in antiken Mythen.

17 Zur „mythischen Gewalt“ als Manifestation göttlicher Macht, welche die Setzung von Recht allenfalls impliziere oder mit sich bringe, nicht aber primär auf Durchsetzung eines bereits bestehenden Rechts in Form einer Strafe abziele, vgl. die Überlegungen von Benjamin (1921=) 1965 (Hinweis Gösta Gabriel). 
(das ancile) zum römischen König Numa, oder der erste von Innana für die Erde gebrachte Tempel (implizit) in die Residenzstadt Uruk.

Dass aus dem Himmel gekommene Objekte für die Gesellschaft wichtig sind, kann sich z. B. darin zeigen, dass sie im Lebensvollzug immer wieder ins Zentrum gerückt werden. So werden die ancilia-Schilde in einem regelmäßig wiederkehrenden Ritual gezeigt und geehrt, oder ein Kultbild wie das Palladion von Troia wird durch Opfergaben und Chorgesänge verehrt; des weiteren werden bspw. Götterbilder durch immer wiederkehrende Mundöffnungsrituale, Tempel durch zyklisch wiederholte Tempelweihfeste in ihrer Wirkmächtigkeit neu ,aufgeladen“. Die Wichtigkeit für eine Ermöglichung, Aufrechterhaltung und Förderung guten menschlichen Lebens wird als Ziel u. a. im Mythos INNANA BRINGT DAS HIMMELSHAUS FÜR DIE ERDE besonders deutlich: Innana schafft durch die Errichtung des ersten Tempels einen Ort, an dem Menschen den Göttern begegnen, ihnen opfern und für ihr eigenes Wohlergehen bitten können, wodurch ein durch die Götter geschütztes (Sicherheit) und damit gutes (Fruchtbarkeit) Leben garantiert werden kann. Daher wird von diesem Himmelshaus gesagt, dass es die Erde „gut macht“.

Es ist nun aber nicht nur so, dass die herausragende Stellung von Örtlichkeiten oder Personen, die Dinge aus dem Himmel empfangen, die Bedeutsamkeit eben dieser Dinge unterstreicht, sondern auch umgekehrt hat die himmlische Herkunft dieser Dinge implizit eine legitimierende Funktion in Hinblick auf die Stellung der empfangenden Orte oder Personen. Denn wer würdig ist, etwas Positives von Göttern aus dem Himmel als Gabe zu erhalten, erhält dadurch die Legitimation für eine herausgehobene Position oder Aufgabe. Dass Innana zur Heiligen Hochzeit aus dem Himmel kommt, legitimiert die Herrschaft von IddinDagan und schenkt ihm und damit dem Land Fruchtbarkeit. Der Besitz der ancilia-Schilde unterstreicht und legitimiert die Position von Numa als einem Herrscher mit einem „besonderen Draht“ zu den Göttern und von Rom als Hauptstadt des römischen Imperiums. Das Palladion ist durch seine himmlische Herkunft nicht nur als außergewöhnlich gekennzeichnet, sondern es trägt durch eben diese Außergewöhnlichkeit auch dazu bei, die herausgehobene Stellung der Königsstadt Troia und ihres Gründers zu legitimieren. Diese legitimierende Funktion, die das Palladion nach griechisch-römischen Quellen durch seinen Ursprung vom Himmel und von den Göttern innehat, kann es weitergeben, wenn es von herrschender Stadt zu herrschender Stadt wandert ${ }^{18}$ - analog zum Königtum,

18 Vgl. Beitrag C. Zgoll, Vom Himmel gefallen. 
das nach mesopotamischen Quellen von herrschender Stadt zu herrschender Stadt wandert ${ }^{19}$.

\subsection{Metafunktionen: kognitive und affektive Stabilisierung}

Welche Auswirkungen können die Erzählungen solcher mythischer Stoffe auf die antiken Menschen gehabt haben, worin bestehen also potenzielle Metafunktionen von Erzählungen über vom Himmel Gekommenes? Im Allgemeinen bewirken mythische Stoffe sowohl eine kognitive als auch eine affektive Stabilisierung, zum einen durch die epistemische Komponente einer orientierungsstiftenden Strukturierung der erfahrenen Wirklichkeit, zum anderen durch psycho-soziale Komponenten wie etwa Gefühle der Orientierung, der Sicherheit und damit der Freiheit von Angst, die auf einer (häufig gemeinschaftlichen) kognitiven Aufarbeitung von Erfahrungsgegenständen beruhen ${ }^{20}$.

So leisten mythische Erzählungen über einen Himmelstransfer („X kommt vom Himmel“) einen wichtigen Beitrag zur kognitiven Verarbeitung von Wirklichkeitserfahrungen, indem sie erklären, woher essenzielle Dinge wie Ordnung, Recht, Sicherheit oder Fruchtbarkeit kommen - und wer „dahintersteht“. Besonders wichtig ist dies nicht nur im Hinblick auf die Erklärung der Provenienz des Guten, sondern gerade auch im Hinblick auf die Herkunft des Schlechten oder Bedrohlichen. Woher das Negative kommt, wird in verschiedenen mythischen Überlieferungen unterschiedlich erklärt, je nach dem Kontext, in dem die Überlieferung steht: Teils ist man nur daran interessiert, den vorliegenden Einzelfall zu erklären, teils soll das Entstehen von Übeln überhaupt oder zumindest für einen bestimmten größeren Bereich erklärt werden, der mehrere ähnliche Fälle umfasst. Der Einzelfall ist gegeben, wo die Erklärung sich nur auf einen speziellen Fall bezieht und nur für einen bestimmten Menschen oder eine Gruppe von Menschen gilt, bei dem bzw. denen ein vorliegendes Übel auf das Wirken einer einzelnen numinosen Macht zurückgeführt wird (eine einzelne Krankheit eines speziellen Menschen oder einer speziellen Gemeinschaft wie etwa eine Seuche, die eine bestimmte Stadt heimsucht). Eine generalisierende Erklärung der Herkunft von Negativem liegt vor, wenn erzählt wird, wie etwas oder jemand vom Himmel auf die Erde gekommen ist, das oder der für die Menschheit insgesamt schlimme Auswirkungen haben konnte wie etwa bei Lamaštu, die wegen ihrer

19 Vgl. Beitrag Gabriel.

20 S. C. Zgoll 2019, 424 f. 
bösen Taten vom Himmelsgott aus dem Himmel geworfen wird (als generelle Verursacherin von Krankheiten ${ }^{21}$ ) oder Ate, die Zeus vom Himmel auf die Erde verbannt (personifizierte Göttin der Verblendung ${ }^{22}$ ).

Bei der Erklärung des Negativen in der Welt wird im Alten Orient nach einer Überlieferung sogar der Irrationalität des „Bösen“ Rechnung getragen: So ist nach dieser Tradition Negatives nicht unbedingt eine notwendige Folge intentionaler Handlungen menschlicher oder göttlicher Akteure, also nicht bspw. Folge menschlichen Fehlverhaltens den Göttern gegenüber oder Folge göttlicher, wie auch immer motivierter Aktionen den Menschen gegenüber, sondern es kann auch auf das Einwirken dämonischer Mächte zurückgeführt werden, die wie mutwillige Marodeure auch dann zerstörerisch tätig werden können, wenn es keinen direkten oder einsichtigen Grund dafür gibt ${ }^{23}$. Dies zeigt bereits der Umstand, dass An Lamaštu aus dem Himmel wirft, denn das impliziert, dass sie vorher gewissermaßen auf eigene Faust, nicht im Auftrag der himmlischen Götter gehandelt hat.

Eminent wichtig für die antiken Kulturen ist nun aber, dass mythische Stoffe nicht nur zur kognitiven, sondern auch zur affektiven Stabilisierung beitragen, dass somit ein Stoff, der von einem Sphärenwechsel aus dem Himmel erzählt, nicht nur die Welt erklärt, sondern dass er zugleich auch Hilfestellungen bietet, die Welt zu bewältigen ${ }^{24}$. Wenn es bspw. um die Frage geht, an wen man sich zu wenden hat, wenn man gute Dinge aus dem Himmel erhalten oder Ungutes abwehren will, dann liegt die Antwort darauf bereits in den jeweiligen Mythen selbst: Man hat sich natürlich am ehesten an diejenigen Gottheiten zu wenden, die nach dem jeweiligen Mythos für die Herabkunft dieser Dinge aus dem Himmel verantwortlich zeichnen, ggf. unter Einschaltung eines Fürsprechers oder Mittlers. Wenn bspw. im antiken Mesopotamien Krankheiten auf das Wirken der Dämonin Lamaštu zurückgeführt werden, dann steckt in dieser Erklärung zugleich

21 Vgl. Beitrag Zomer.

22 Vgl. den Beitrag C. Zgoll, Göttergaben und Götterstürze.

23 Vgl. auch van Binsbergen/Wiggermann 1999, 27 zur teils in Mesopotamien sichtbaren Vorstellung von Dämonen als ,amoral outsiders sharing neither the burdens nor the profits of civilisation“. Bottéro 2001, 186 f spricht von einer „magischen Erklärung“, wenn böse Dämonen dem Menschen grundlos, aus reiner Bosheit schaden.

24 S. dazu C. Zgoll 2019, 425 f. Die Betonung speziell der Angstbewältigung ist außerdem ein zentrales Anliegen in Blumenbergs Monographie Arbeit am Mythos (1979), der dadurch wesentlich dazu beigetragen hat, innerhalb der Mythosforschung die allzu einseitige Fixierung auf den kognitiven Aspekt der Funktionalisierung mythischer Stoffe (vgl. bspw. Beth 1935, 721) aufgebrochen zu haben. 
auch schon die Möglichkeit, die Krankheit zu besiegen, indem man nämlich genau diese Dämonin wieder vertreibt. Und wie das geschehen kann, dafür wird in der altorientalischen Mythologie wiederum ein mythisches Narrativ zur Verfügung gestellt: Es handelt von Asalluhi, dem numinosen Antagonisten der Lamaštu, und davon, wie dieser im mythischen Einst Lamaštu überwunden und vertrieben hat; so kann und soll dann auch im historischen Jetzt Lamaštu durch Herbeirufen und mit Hilfe von Asalluhi vertrieben werden ${ }^{25}$.

\section{Von der Funktionsanalyse zur Interpretation}

\subsection{Vertiefung 1: Anzeiger für Prototypisches}

Mythen über eine Herkunft aus dem Himmel erzählen davon, dass etwas aus dem himmlischen Bereich herabkommt, in den meisten Fällen auf die Erde und damit $\mathrm{zu}$ den Menschen. Ist diese Aussage noch weitgehend trivial, so ist die Feststellung, dass es sich dabei stets um etwas für die damaligen Kulturen besonders Bedeutsames handelt, schon deutlich pointierter und für die Interpretation dieser Mythen folgenreicher. Bei näherer Betrachtung lässt sich dies inhaltlich weiter zuspitzen, insofern solche mythischen Erzählstoffe oft auf etwas abzielen, was für die Interpretation dieser Mythen ebenfalls einen wichtigen Faktor darstellt, auch wenn es nicht bei all solchen Erzählstoffen aufs Erste erkennbar ist. Diese Zuspitzung besteht darin, dass Gegenstände, Kulturtechniken oder Einrichtungen, die aus dem Himmel kommen, in der Regel etwas Prototypisches bezeichnen $^{26}$.

Deutlich ist dies noch bspw. bei den mesopotamischen Mythenvarianten zu erkennen, die von der HIMMLISCHEN HERKUNFT DES KÖNIGTUMS berichten ${ }^{27}$. Wenn das Königtum aus dem Himmel kommt, dann hat es so etwas vorher auf Erden nicht gegeben, dann ist das eine Himmelsgabe für die Menschen, die so das erste Mal erfolgt und erst ab diesem Zeitpunkt auf Erden besteht. Ähnlich liegt der Fall bei der Herabkunft des ersten Tempels aus dem Himmel. Wenn Innana das E-ana, das „Haus des Himmels“ aus dem Himmel herabbringt, dann zielt dies nicht nur auf einen Tempel in Uruk, sondern auf den ersten Tempel überhaupt, was noch

25 So die Ergebnisse der Forschungen von Brit Kärger, die eine ausführlichere Studie dazu vorbereitet.

26 Dies gilt nicht unbedingt auch für numinose Wesen, die vom Himmel kommen.

27 Vgl. Beitrag Gabriel. 
um so deutlicher wird, wenn man der Argumentation folgt, dass mit der Begründung dieses Tempels zugleich die Gründung Sumers und der Erde vollzogen wird $^{28}$. Und wenn Prometheus den Menschen das Feuer bringt, dann ist dies etwas Neues, Erstmaliges, wofür der Protagonist nach manchen Stoffvarianten schlimmste Qualen auf sich nehmen muss ${ }^{29}$.

Ist in diesen Mythen klar erkennbar, dass sich hier „Erstereignisse“ vollziehen und etwas Prototypisches erstmalig ins Sein gerufen wird, so liegt dies in etlichen anderen Fällen nicht gleichermaßen klar zutage. Das kann verschiedene Gründe haben. Zum einen kann dies daran liegen, dass man das Prototypische als bekannt vorausgesetzt hat und daher keine Veranlassung sah, diesen Aspekt explizit zu erwähnen. Damit hängt zusammen, dass mythische Stoffe oft sehr abgekürzt überliefert worden sind, und gerade deswegen oft das, was als selbstverständlich galt, in der Verkürzung weggelassen werden konnte ${ }^{30}$. Ein weiterer Grund kann darin bestehen, dass verschiedene Überlieferungen miteinander in Konkurrenz getreten sind. Warum dies zur Weglassung der Betonung des Prototypischen führen kann, soll im Folgenden deutlich werden.

\subsubsection{Konfliktträchtigkeit von Prototypen-Mythen}

Aufgrund der bereits lange andauernden schriftlichen und vor allem mündlichen Überlieferung und vor allem aufgrund zahlreicher inner- und transkultureller Kontakte, also aufgrund des Phänomens der Interhylität ${ }^{31}$, stehen verschiedene mythische Stoffe und ihre Varianten, in unserem Fall verschiedene Mythen über eine himmlische Herkunft z.T. derselben Gegenstände, Kulturtechniken oder Einrichtungen, oft unvermeidlich miteinander in einer gewissen Konkurrenz. Gerade der eben angesprochene Fall des E-ana-Tempels, der als erstes „Himmelshaus“ von Innana aus dem Himmel herabgeholt wurde, kann deutlich machen,

28 Vgl. Beitrag A. Zgoll, Wie der erste Tempel auf die Erde kommt.

29 Vgl. Beitrag Alvensleben.

30 Vgl. dazu C. Zgoll 2019, 197-204 und die Untersuchungen von Kärger in diesem Band zu vielen isoliert überlieferten mythischen Hylemen in der mesopotamischen Literatur. Vgl. auch A. Zgoll 2020 zur Funktion eines solchen isolierten Hylems innerhalb eines anderen Erzählstoffes. Es handelt sich dort um ein isoliertes Hylem, das den mythischen Erzählstoff von INNANA BRINGT DAS HIMMELSHAUS FÜR DIE ERDE zusammenfasst; durch seine Parallelisierung mit einem anderen isolierten Hylem, welches auf den Mythos INNANA BRINGT MACHTMITTEL DER UNTERWELT ZUR ERDE rekurriert, wird das isolierte Hylem des einen Mythos zum Schlüssel für die Bewertung und den Ausgang des anderen Mythos eingesetzt.

31 Zum Phänomen der Interhylität s. ausführlich C. Zgoll 2019, 270-288. 
was geschehen kann, wenn mehrere mythische Stoffe zu demselben Thema miteinander (in diesem Fall innerkulturell) in Kontakt treten. Der Mythos vom E-ana verweist auf Uruk, und damit auf eine besondere Lokaltradition. In der Stadt Eridu z. B. wurde aber ein ganz ähnlicher mythischer Stoff erzählt, dort allerdings auf den Gott Enki bezogen, der den ersten Tempel aus dem unterirdischen Süßwasserozean Abzu heraus dort entstehen lässt, wo die Stadt Eridu liegt. Da Mythen aus nah beieinander liegenden Stadtstaaten nicht isoliert voneinander bleiben, können, ja müssen fast zwangsläufig zwei verschiedene, eigentlich unvereinbare mythische Stoffe über die Entstehung des ersten Tempels auf Erden miteinander in Konflikt geraten.

Ein Mittel, um diesen Konflikt zu entschärfen, besteht aus einem relativ geringfügigen Eingriff: indem man nämlich die explizite Zuspitzung, dass es sich jeweils um den ersten Tempel überhaupt gehandelt hat, abschleift bzw. auslässt. Stratifikationsanalytisch betrachtet ist in diesen Fällen somit das Fehlen eines Stoffzuges, in diesem Fall das Fehlen einer expliziten Kennzeichnung als Erstereignis, bereits ein Indiz für eine Stoffschichtung, die auf ausgetragene Deutungsmachtkonflikte hindeuten kann. Denn es ist mehr als plausibel, dass die ursprünglich selbständigen sumerischen Stadtstaaten ihre jeweils eigenen Traditionen entwickelt haben, und dass damit die Bewohner von Eridu in Enkis Haupttempel genauso den ersten Tempel überhaupt erblickt haben wie die Einwohner von Uruk in der Heldentat der Innana, die das E-ana aus dem Himmel gebracht hat, eine Tat sahen, die eine Erst-Tat war.

Geht man von diesen Beobachtungen aus, dann legt es sich nahe, dass auch in Überlieferungen anderer Kulturen und Zeiten Himmelsgaben in vielen Fällen als Erst-Gaben zu interpretieren sind, auch wenn aufgrund der Konkurrenz ähnlicher Mythen (oder weil man es als selbstverständlich voraussetzte) diese Gegenstände, Kulturtechniken oder Einrichtungen nicht immer explizit als etwas Prototypisches gekennzeichnet sind. So ist etwa in Griechenland die Gabe des Feuers durch Prometheus z.B. in Hesiods Theogonie noch eindeutig als Erst-Gabe gekennzeichnet ${ }^{32}$; in anderen Fällen aber wird die Frage diffiziler und damit in Hinblick auf die Mytheninterpretation spannender. Dies soll an einem weiteren Beispiel aus der griechischen Mythologie näher verdeutlicht werden.

32 Vgl. Beitrag Alvensleben. 


\subsubsection{Beispiel Palladion: Die Himmelsgabe des Palladions als mythisches Erstereignis}

In vielen Fällen, so hat sich gezeigt, zielen Mythen über eine himmlische Herkunft auf etwas Prototypisches, und zwar auch dann, wenn dies nicht (mehr) explizit ausgedrückt wird. Das hat zur Folge, dass es sinnvoll, wenn nicht notwendig ist, sich bei der Interpretation solcher Mythen die Frage zu stellen, ob eine Himmelsgabe eine Erst-Gabe darstellt, und zwar auch und gerade dann, wenn dies eben nicht explizit vermerkt ist. Ein solcher Fall liegt bspw. bei der himmlischen Gabe des Palladions bzw. der Palladia vor ${ }^{33}$. Wie sind die diesbezüglichen mythischen Stoffvarianten hinsichtlich der Frage nach etwas Prototypischem zu interpretieren?

Ob nun nach der mythischen Überlieferung der Griechen Athene der Chryse die Palladia als Hochzeitsgeschenk überreicht, oder ob Zeus ein Palladion zu Ilos aus dem Himmel herabfallen lässt oder dem Dardanos übergibt, so handelt es sich bei diesen Gaben jeweils mit Sicherheit um etwas Besonderes, denn Götter geben, wenn sie geben, nichts „Normales“, sondern etwas Außergewöhnliches ${ }^{34}$. Nun gibt es auch hier prinzipiell zwei Möglichkeiten: Entweder handelt es sich beim Palladion um eine außerordentliche Gabe, die auf Erden so oder in vergleichbarer Form bereits existiert hat, oder die Außergewöhnlichkeit dieser Gabe besteht nicht zuletzt darin, dass es so etwas oder auch Vergleichbares bis dahin nicht gegeben hat.

Eine für die troianische Lokalüberlieferung ausnehmend wichtige Rolle des Palladions in seiner Funktion als ein das Heil der Stadt garantierendes Götterbild kann kaum bestritten werden. Kann man eventuell aber auch so weit gehen, dass mit diesen mythischen Stoffvarianten implizit die Vorstellung verbunden war, dass die Menschen überhaupt erst seit dem Geschenk des Palladions (bzw. der Palladia) Götterbilder kannten, die unter anderem die ausnehmend wichtige Funktion hatten, den Schutz ihrer Städte zu gewährleisten?

Dass es nach der mythischen Weltsicht der genannten griechischen Stoffvarianten noch keinerlei kultische Repräsentationen von Göttern auf Erden gegeben haben könnte, bevor das Palladion den Menschen zum Geschenk gemacht wurde, lässt sich nicht zwingend ableiten. Schon etwas anderes ist es hingegen mit der Zuspitzung, dass es nach den überlieferten PALLADION-MYTHEN vor der Gabe des Palladions auf Erden noch keine Kultbilder gegeben haben könnte, die

33 Vgl. Beitrag C. Zgoll, Vom Himmel gefallen.

$34 \mathrm{Zu}$ Göttergaben generell s. auch den Überblicks-Beitrag C. Zgoll, Göttergaben und Götterstür$z e$ in diesem Band. 
speziell für die Sicherheit einer befestigten Stadt von höchster Wichtigkeit waren und demgemäß gehütet und verehrt wurden. Wenn mythische Stoffe über eine himmlische Herkunft, wie oben gezeigt, oft von Erstereignissen handeln oder Prototypisches vor Augen führen, kann es sogar als wahrscheinlich gelten, dass die bspw. bei Apollodoros vorgestellte Stoffvariante impliziert, dass Zeus mit seiner Gabe des Palladions an Ilos anlässlich der Stadtgründung von Troia tatsächlich erstmals einen solchermaßen spezifizierten Kultgegenstand auf die Erde gelangen lässt ${ }^{35}$. Selbst wenn dem nicht so wäre, muss man noch den Umstand berücksichtigen, dass es mehrere verschiedene Lokaltraditionen gegeben hat, für die jeweils aus ihrer Sicht ein „First“ behauptet worden sein kann. Das wird erst dann problematisch, wenn man der Versuchung erliegt, diese verschiedenen Lokaltraditionen zu einer „Gesamtmythologie der Griechen“ zu synthetisieren und $\mathrm{zu}$ harmonisieren (vgl. analog oben die Diskussion zum ersten Tempel in Mesopotamien und $\mathrm{zu}$ den diesbezüglich unterschiedlichen Lokaltraditionen von Uruk und Eridu).

Zusammenfassend lässt sich sagen, dass in (auserzählten, nicht in abgekürzten) mythischen Stoffen (bzw. ihren Varianten) von einer himmlischen Herkunft bei näherer Betrachtung nicht allgemein von Objekten in einem generischen Sinn die Rede ist, also bspw. von der Herabkunft von Tempeln überhaupt oder von Götterbildern generell, welche die Funktion haben, eine Stadt zu schützen, sondern dass Entsprechendes in aller Regel an einem spezifischen Objekt veranschaulicht wird, das stellvertretend, aber eben konkret, für eine ganze (abstrakte) Klasse steht. Das konkrete Palladion steht somit stellvertretend für die „Klasse“ von Götterbildern mit Schutzfunktion für eine Stadt, und wenn nach mythischer Überlieferung spezifisch das Palladion von Troia erstmals vom Himmel auf die Erde kommt, dann kann das für die Interpretation entsprechender Mythenvarianten bedeuten, dass in deren Sicht mit diesem Palladion auch erstmals überhaupt ein städteschützendes Götterbild zu den Menschen gelangt ist, auch wenn dies nicht (mehr) explizit zum Ausdruck gebracht wird.

Diese Schlussfolgerungen werfen schließlich noch einmal ein neues Licht auf das Problem, dass die antiken Überlieferungen schwanken, ob aus dem Himmel bzw. von bestimmten Göttern ein Palladion gegeben wurde oder mehrere Palladia gebracht worden sind ${ }^{36}$ (vgl. analog den strukturell ganz ähnlichen, römischen Fall von dem einen ancile bzw. mehreren ancilia-Schilden ${ }^{37}$ ). In der griechisch-römischen Antike selbst hat man dies entweder damit erklärt, dass es

35 Apollod. 3,143.

36 S. dazu den Beitrag C. Zgoll, Vom Himmel gefallen, Abschnitt 7 in diesem Band.

37 Vgl. Beitrag Jäger. 
von Anfang an mehrere Palladia waren, oder damit, dass es nur eines gab, dass man aber, um dieses eine wertvolle Palladion zu schützen, mehrere Kopien angefertigt habe. Oder man versucht das Problem diachron zu lösen, indem man annimmt, dass es nur ein Palladion gegeben hat, welches dann aber, wie das nur einmal vom Himmel herabgekommene Königtum in Mesopotamien ${ }^{38}$, von Stadt zu Stadt „wanderte“.

In gewisser Weise verdeutlichen aber gerade die beiden zuletzt genannten Lösungen vom Kopieren und vom Wandern, dass es eigentlich nur ein wahres, aus dem Himmel gekommenes Palladion gegeben hat und geben kann, denn sie entpuppen sich in ihrer Konstruiertheit deutlich als Notlösungen bzw. Kompromisse. Die Lösung, dass Götter aus dem Himmel eine bestimmte einzelne Person (sei es nun Chryse oder Dardanos oder Ilos) mit mehreren Palladia beschenken, stellt deutlich erkennbar den Versuch dar, dem Befund mehrerer verschiedener Palladion-Traditionen gerecht zu werden und etwas für Mythen über eine himmlische Herkunft eher Untypisches, Künstliches zu konstruieren, indem eine einzelne, konkrete Person gleich eine ganze Gruppe von Gegenständen (,die Palladia“) erhält. Prototypen-Mythen hingegen zielen in der Regel darauf, dass einer einzelnen, konkreten Person passenderweise auch eine einzelne, konkrete Gabe zuteil wird, die dann auch zum ersten Mal überhaupt in den menschlichen Bereich gelangt.

\subsection{Vertiefung 2: Anzeiger für Numinosität}

Die Erzählung des Vorgangs „X kommt vom Himmel (zu/nach Y)“ kommt für vielfältige Einsatzmöglichkeiten in Frage und hat sich daher als ein wirkmächtiger Erzählbaustein in antiken Mythen verschiedener Kulturen erwiesen. Neben der Funktionalisierung als Anzeige von etwas Prototypischem ist noch eine weitere Funktionalisierungsmöglichkeit all diesen unterschiedlichen Konkretionen des genannten Vorgangs gemeinsam. Diese wird ebenfalls erst deutlich, wenn man einen Schritt zurücktritt und auf eine abstraktere Aussage-Ebene fokussiert, die hinter den so unterschiedlich ausgestalteten Erzähloberflächen liegt. Und auf dieser Ebene wird immer dieselbe Botschaft transportiert: Dass nämlich das, was vom Himmel kommt, von den Göttern kommt und dementsprechend Göttlichkeit besitzt. Die eigentliche Pointe liegt nicht auf dem Ort der Herkunft all dieser Dinge und Figuren, sondern darauf, dass dieser Ort auf Göttlichkeit oder allgemeiner:

38 S. dazu den Beitrag Gabriel, Abschnitt 3.3 in diesem Band. 
auf Numinosität dessen deutet, was von diesem Ort kommt ${ }^{39}$. Der Himmel ist in gewisser Weise austauschbar, da sowohl Positives wie Negatives teilweise auch aus anderen kosmischen Bereichen kommen kann; nicht austauschbar aber ist die Stoßrichtung der Aussage, dass es sich bei diesen Dingen oder Gestalten um etwas Numinoses handelt.

Diese Erkenntnis hat wichtige Auswirkungen auf die Interpretation einzelner Mythen. Als Beispiel greifen wir zwei Mythen heraus, einen aus der griechischen, einen aus der sumerischen Überlieferung: den Mythos vom FEUERDIEBSTAHL DES Prometheus ${ }^{40}$ (unter 3.2.2) und einen Mythos, der von einem sumerischen Helden namens Lugalbanda handelt ${ }^{41}$.

\subsubsection{Beispiel Lugalbanda: Die göttliche Legitimierung des Heerführers Lugalbanda als neuer Herrscher}

Zunächst zum sumerisch überlieferten Erzählstoff von Lugalbanda. Ein großer epischer Text schildert den Feldzug des sagenhaften Urzeit-Herrschers Enmerkara von Uruk zum ferne in den östlichen Bergen gelegenen Arata ${ }^{42}$. Dieses sog. Lugalbanda-Epos ist auf Tontafeln aus den Jahrhunderten kurz vor und nach 2000 v. Chr. erhalten ${ }^{43}$.

39 Als „numinos“ werden im vorliegenden Auswertungsbeitrag Wesen und Phänomene und deren Einwirkungen auf Geschehnisse bezeichnet, die in emischer Sichtweise die menschliche Natur und deren Fähigkeiten transzendieren (vgl. C. Zgoll 2019, 395; dort in Bezug auf die transzendierende Auseinandersetzung mit Erfahrungsgegenständen, die ein wesentliches Merkmal mythischer Stoffe darstellt), außerdem Örtlichkeiten, die vornehmlich von solchermaßen charakterisierten Wesen bewohnt oder beherrscht werden. Dieser Kategorie des Numinosen gehören bspw. Wesen wie Götter oder Dämonen oder Orte wie Himmel oder Unterwelt an, daneben auch Gegenstände oder Handlungen (z. B. Kultgegenstände oder Rituale), die in einem Zusammenhang mit numinosen Wesen stehen. Zur Rechtfertigung der Kategorie des Numinosen in der Konzeption von Rudolf Otto (als mysterium tremendum et fascinans) aus religionswissenschaftlicher Sicht, zu ihrer Valenz für eine kulturwissenschaftliche Analyse und zu ihrer Verteidigung gegen Kritik s. die Arbeit von Johannsen 2008.

40 Zum Mythos Prometheus STIEHLt DAS FeUER und seinen verschiedenen Varianten in der Überlieferung s. den Beitrag von Alvensleben im vorliegenden Band.

$41 \mathrm{Zu}$ Lugalbanda, der erscheint wie einer, der vom Himmel kommt, s. den Beitrag von Kärger im vorliegenden Band (v. a. Abschnitt 4.2.4.1).

42 Aus heutiger Perspektive betrachtet geht es um den Feldzug eines Stadtstaates im Irak gegen einen Stadtstaat im Iran.

43 Claus Wilcke hat den zweiten Teil dieses Epos ediert (Wilcke 1969), die Zusammengehörigkeit beider Teile erkannt und als Einheit neu übersetzt (Wilcke 2015) sowie die zentralen Erzählstoffe erschlossen (Wilcke 2015, 208-211). Wilcke spricht hier von Geschichten: „Drei Geschichten 
Gemeinsam mit Enmerkara sind sieben Generäle an dem Feldzug gegen Arata beteiligt, außerdem ein Achter, der namentlich hervorgehoben wird, Lugalbanda. Auf dem Weg ins Gebirge wird Lugalbanda todkrank und muss im kur, dem „Gebirge“ bzw. „Totenreich“ (beide Bedeutungen stecken im sumerischen Wort) zurückgelassen werden. Durch göttliche Hilfe gelingt es Lugalbanda, zu Enmerkara und den Truppen zurückzukehren. Diese Rückkehr aus dem Totenreich wird durch verschiedene Vergleiche als etwas Erstaunliches und Unfassbares markiert. Hier findet sich auch der entscheidende Verweis auf das Hervorkommen aus dem Himmel. Lugalbanda kommt nämlich zu seinen Brüdern wie eine Person, die vom Himmel kommt:

${ }^{222} \mathrm{lu}_{2}$ an-ta ki-a gub-ba-gen 7 ${ }^{223}$ lugal-ban 3 -da $z u_{2}-k e \check{s}_{2} \hat{g}$ arra šeš-a-ne-ne-ka murub ${ }_{4}-b a$ ba-an-gub?
${ }^{222}$ Wie jemand, der sich aus dem Himmel kommend auf der Erde aufgestellt hat, ${ }^{223}$ stellte sich Lugalbanda in die Mitte der aufgestellten Truppen seiner Brüder. ${ }^{44}$

Lugalbanda-Epos Teil 2, Z. 222

Ein Hinweis auf eine Herkunft vom Himmel ist textintern auch an anderer Stelle des Epos zu finden ${ }^{45}$. Es handelt sich dort um den Herrscher Enmerkara, der sich aus dem Himmel kommend in der „Großen Erde“ (= Unterwelt; s. dazu unten) aufgestellt hat. Betrachtet man die beiden Schilderungen des Urkönigs Enmerkara und des Heerführers Lugalbanda in Bezug auf die Herkunft vom Himmel, fällt ein entscheidender Unterschied auf: Während Enmerkara durch die Aussage, dass er vom Himmel kommt, direkt als göttlich charakterisiert wird (Teil 1, Z. 53; s. unten), wird Lugalbanda (Teil 2, Z. 222) nur mit einem göttlichen, vom Himmel kommenden Wesen verglichen.

Die Frage ist, was hinter dieser Differenzierung steckt. Sie ist verbunden mit dem Problem, weshalb ein militärischer Anführer, und sei es auch nur vergleichsweise, als göttlich beschrieben wird, wenn Numinosität ansonsten nur bestimmten geweihten Herrschern zukommt. Wesen, die sich vom Himmel kommend auf

in einer?“ (a. 0. 208); dem entspricht in der Terminologie der Stoffwissenschaft (Hylistik), dass in einem Text drei Erzählstoffe verarbeitet sind. - Claus Wilcke hat uns großzügig ermöglicht, das unpublizierte Manuskript seiner Partitur des Lugalbanda-Epos von 2011 zu verwenden.

44 Vgl. den Beitrag von Kärger im vorliegenden Band unter Abschnitt 4.2.4.1 und Anhang 33 sowie Wilcke 2015, 263.

45 Claus Wilcke ist der Nachweis gelungen, dass es sich bei den früher als zwei Epen angesehenen Texten vielmehr um ein einziges episches Preislied handelt, vgl. Wilcke 2015, $207 \mathrm{f}$. 
der Erde aufstellen, sind nach den Ergebnissen des Beitrages von Brit Kärger in Teil 2 dieses Bandes klar als Gottheiten markiert. Lugalbanda erscheint seinen „Brüdern“, den anderen Generälen, hier mithin wie eine Gottheit. Dass ein König als gottgleich angesehen wird, ist für Mesopotamien gut bezeugt. Etliche Herrscher seit Narām-Sîn (23. Jh. v. Chr.) bezeichnen sich als Gottheit und schreiben ihren Namen mit dem Gottesdeterminativ. Aber wieso Lugalbanda? Lugalbanda ist kein Herrscher. Er ist vielmehr der achte Heerführer des Enmerkara. Offensichtlich kann die Numinosität also nicht vom Amt des Heerführers abgleitet werden, im Gegenteil: Die übrigen Feldherren sind schockiert wie bei einer echten Theophanie (Teil 2 Z. 220-224) und wundern sich über die Ausstrahlung, die ihren „Bruder“ auszeichnet (Teil 2, Z. 220-237).

Weitere Eigentümlichkeiten fallen auf: (1) Obwohl das Epos mit dem Auszug gegen Arata anhebt, endet es nicht mit dem Sieg über diese Stadt, wie man das üblicherweise erwarten würde. (2) Obwohl der Herrscher, der am Anfang preisend eingeführt wird, der berühmte Enmerkara ist, über den auch andere Preislieder erhalten sind, endet das epische Lied nicht mit einem Preis des Enmerkara. Vielmehr steuert der Text auf den Preis des achten militärischen Anführers, eben des Lugalbanda zu.

Hier erweist sich einmal mehr der Gewinn der Rekonstruktion mythischer Erzählstoffe durch Hylemanalyse, da diese vom Text, der bewusst gewisse Härten zu mildern sucht, zu den „narrativen Fakten“ führt, also den Erzählbausteinen, die auf der Textoberfläche teils verschleiert, teils umgestellt, teils ausgespart werden ${ }^{46}$. Welche „gewisse Härten“ sind hier gemeint? Im Folgenden soll plausibel gemacht werden, dass der eigentliche Kernpunkt des Lugalbanda-Epos nicht darin besteht, einen siegreichen Feldzug gegen ein feindliches Volk in den Bergen $\mathrm{zu}$ beschreiben, sondern zu erklären, wie es im Verlauf dieses Feldzuges zu einem Machtwechsel zwischen dem legitimen Herrscher Enmerkara und dessen Heerführer Lugalbanda gekommen ist, und diesen Herrschaftswechsel zu rechtfertigen und göttlich zu legitimieren. Einer, wenn nicht der zentrale mythische Erzählstoff, der dem Lugalbanda-Epos zugrundeliegt, ist somit der Mythos DIE GÖTTER MACHEN LUGALBANDA ZUM HERRSCHER. Die Rekonstruktion dieses mythischen Erzählstoffes ist komplex, da der Text außergewöhnlich umfangreich ist; hier können daher nur einige wesentliche Teile herausgegriffen werden ${ }^{47}$.

Der menschliche Protagonist in diesem mythischen Erzählstoff DIE GÖTTER MACHEN LUGALBANDA ZUM HERRSCHER ist nach dem eben Ausgeführten nicht, wie sonst üblich, der aktuell amtierende Herrscher selbst, Enmerkara, sondern eben

46 Vgl. C. Zgoll, Grundlagen der hylistischen Mythosforschung, Abschnitt 2.8, in diesem Band. 47 Vgl. Wilcke $2015 \mathrm{zu}$ anderen Erzählstoffen, die im Text verarbeitet sind. 
sein Heerführer Lugalbanda. Dieser Lugalbanda wird außergewöhnlich aufgewertet; in diese Richtung weisen bereits die Beobachtungen von Claus Wilcke: Lugalbandas „Abenteuer ... und seine Entwicklung zu einem künftigen Herrscher stehen fast durchweg im Vordergrund der Erzählung“48. Der eigentliche Herrscher Enmerkara hingegen muss letztlich sein königliches Amt abgeben (siehe unten). Das wird allerdings so vorsichtig angedeutet, dass deutlich wird, dass gerade hierin ein großes Problem liegt. Dieses Problem wird verständlich, wenn man sich die Situation unbeeinflusst vom Wissen um sonstige Erzählstoffe über Lugalbanda oder Enmerkara vor Augen hält ${ }^{49}$. Bei näherer Betrachtung wird deutlich: Dieser Lugalbanda erhält noch zu Lebzeiten, also während der Regierungszeit Enmerkaras die Herrschaft übertragen. Man könnte das noch härter und zugespitzter so formulieren: Noch während Enmerkara die Regierung innehat, übernimmt Lugalbanda als Usurpator die Macht ${ }^{50}$.

Dass eine solche Thematik alles andere als einfach zu behandeln ist, liegt auf der Hand. Alles kann nur mit äußerster Delikatesse angedeutet werden. Der stärkste Eingriff des Textes in den Erzählstoff besteht darin, dass das Ende des Mythos in dieser epischen Konkretion überhaupt nicht erzählt wird, zumindest nicht an der zu erwartenden Stelle, am Ende des Textes. Hier ist gerade nichts von einer Thronbesteigung Lugalbandas zu vernehmen. Und so bleibt zumindest an dieser Stelle im Text (!) versöhnlich ausgespart, dass der Mythos, den der Text bearbeitet, von der Inthronisation Lugalbandas handelt, während der bisherige Amtsinhaber noch lebt und noch als König seine Truppen im Feindland befehligt $\mathrm{t}^{51}$.

In einer Situation, wo der amtierende König Enmerkara erfolglos und weit entfernt im iranischen Bergland mit der Belagerung einer fremden Stadt befasst ist, kehrt Lugalbanda - im Auftrag des Königs, wie es heißt - in die Hauptstadt zurück. Hier in der Hauptstadt erwählt ihn die Göttin Innana als ihren Gemahl (Teil 2, Z. 345-355). Das kann nicht anders interpretiert werden, als dass Lugalbanda hier zum neuen, rechtmäßigen Herrscher bestimmt wird. Auf Textebene

\footnotetext{
48 Wilcke 2015, 208.
}

49 Lugalbanda ist sonst vielfach auch als Gottheit bekannt; dies ist teils auch in Handschriften des Lugalbanda-Epos eingedrungen. Enmerkara ist Protagonist und Held in zwei weiteren epischen Dichtungen, Enmerkara und Ensuhkešana, Edition Wilcke 2012, und Enmerkara und der Herr von Arata, Edition Mittermayer 2009.

50 Zur Diskussion der etischen Deutung des Vorgangs als Usurpation s. unten.

51 Gerade diese Abweichung der Textgestaltung vom verarbeiteten Erzählstoff führt dazu, dass das eigentliche Ende des Mythos (dazu unten) nicht wahrgenommen wird, was zu abwegigen Interpretationen führen kann (wie bei Hawthorn 2020, wo auch die neuesten Forschungsergebnisse von Wilcke 2015 nicht rezipiert sind). 
ist dies vorsichtig, aber doch eindeutig formuliert: Innana richtet ihren Blick auf Lugalbanda wie auf ihren Ama-ušumgal-ana. Insofern Ama-ušumgal-ana eine der Bezeichnungen von Innanas Geliebtem und Ehemann ist, läuft das auf die Aussage hinaus, dass Innana Lugalbanda zu ihrem Ehemann nimmt.

Ein solches Geschehen ist aus rituell-mythischen Liedern von etwa $2000 \mathrm{v}$. Chr. gut bezeugt. Es handelt sich um ein Erwählungsritual durch Innana, das modern auch mit dem Namen „Heilige Hochzeit“ bezeichnet wird ${ }^{52}$. Der Bezug ist eindeutig auch dadurch, dass Lugalbanda vor die Göttin Innana tritt, während diese ihn auf ihrem kostbaren Lager erwartet (Teil 2, Z. 348) ${ }^{53}$.

Neben dem Ritual der Heiligen Hochzeit wird im mythischen Text auf ein weiteres Ritual angespielt, das für den Erhalt der königlichen Macht wichtig ist. Und zwar deutet der Herrscher Enmerkara in metaphorischer Redeweise auf ein Ritual, das dazu dient, dass „die königliche Macht erneuert werden kann“54. Der König muss hierfür lebendig in ein Grab steigen und eine Zeit in diesem Grab, d. h. in der Unterwelt verbringen, um durch den Durchgang durch das Totenreich mit neuer Macht zurückzukehren ${ }^{55}$. Ein entsprechender Ritualtext ist aus dem 1. Jahrtausend bezeugt, demzufolge ein solches Ritual jährlich stattfindet ${ }^{56}$. Enmerkara spielt auf dieses Ritual an und macht Innana zum Vorwurf, dass sie ihn

52 Für den vorliegenden Kontext vgl. Wilcke 2015, 213 f. - Bestimmte Rituale wie die Heilige Hochzeit oder das unten besprochene Ritual des königlichen Unterweltsgangs sind äußerst sensible Vorgänge, schon allein deswegen, da eine Störung solcher Rituale in originärer Perspektive unabsehbare Folgen hätte zeitigen können; solche Rituale sind integraler Bestandteil von größeren Ritual- und Festkontexten wie Inthronisations- und Neujahrfesten. Es ist nicht überraschend, sondern zu erwarten, dass derart exklusive heilige Handlungen in profanen Urkunden aus dem Alltags- und Wirtschaftsleben kaum oder gar nicht explizit erwähnt werden. Selbst in Ritualtexten des 1. Jahrtausends sind sie selten und nur in Andeutungen zu finden; immerhin gibt es Ausnahmen wie etwa Zeugnisse zum Ritual vom königlichen Unterweltsgang, vgl. Ambos 2008, 82-85 und Ambos 2008a. Anspielungen auf das Ritual der Heiligen Hochzeit sind in Ritualtexten des 1. Jahrtausends kaum zu erwarten, da dieses Ritual nach bisherigem Kenntnisstand etwa 1000 Jahre früher praktiziert wurde. Ältere Quellen vom Anfang des 2. Jahrtausends haben aber durchaus Teile der rituellen Praxis überliefert wie ein Kultlied auf Innana in ihrer astralen Erscheinungsform, das beim Ritual der Heiligen Hochzeit Innanas mit dem Herrscher Iddin-Dagan von Isin eingesetzt wurde (Iddin-Dagan A, Black et al. 2006c, Attinger 2014), außerdem Anspielungen wie im Lugalbanda-Epos und in der Enmerkara-Literatur.

53 Wilcke 2015, 269. Dass Lugalbanda in Ritualen erfahren ist, wird auf Textebene gleich bei seiner Einführung betont. Er führt Reinigungsrituale durch und „königliche Opfer“ (Lugalbanda-Epos Teil 1 Z. 72, 74).

54 Wilcke 2015, 223.

55 Vgl. Wilcke 2012, 23-25 und Wilcke 2015, 223 f. mit Enmerkara und der Herr von Arata 61 f. // 92 f., Enmerkara und Ensuhkešana 95-98.

56 Vgl. Ambos 2008, 82-85 und Ambos 2008a. 
gerade nicht so behandelt, wie es nach diesem Ritual zu erwarten wäre, dass sie ihn nämlich nicht zurückgebracht hat in ihren Tempel (Z. 315-318 // Z. 379-382). Schon relativ früh im Lugalbanda-Epos gibt es einen ersten Bezug auf dieses Ritual - der genau im Kontext der Herkunft vom Himmel steht. Die Stelle lautet ${ }^{57}$ :

${ }^{54}$ an-ta ki gal-la gub-ba-am 3

${ }^{54} \operatorname{Er}$ (= Enmerkara) war es, der sich aus dem Himmel herauskommend in der Großen Erde (= Unterwelt) ${ }^{58}$ aufgestellt hatte.

Lugalbanda-Epos Teil 1, Z. 54

Die Formulierung mit dem Gegensatzpaar Himmel und Erde und der Bewegung vom Himmel herab in die Große Erde (= Unterwelt) verbindet die Stelle intertextuell mit dem antik gut bekannten Preislied Innanas Gang zur Unterwelt. Dort heißt es „Vom Großen Himmel auf die Große Erde richtete jemand/eine Gottheit/Innana (ihr Ohr =) ihre Planungskraft (und das hatte Konsequenzen) ${ }^{* 59}$. Durch seinen Durchgang durch das Totenreich ist das königliche Ritual, das Enmerkara durchführt, als spezifisches Innana-Ritual gekennzeichnet. Der Hintergrund ist folgender: Gerade die Göttin selbst wird ja gerühmt für ihren Durchgang durch das Totenreich; und gerade bei ihr führt dieser Durchgang durch Sterben und Tod zur höchsten Macht, zur Macht über den Tod und über das Leben (A. Zgoll 2020a). Hier deutet sich schon an, was sich noch näher zeigen wird: Dieser Gang ins Totenreich ist ein Rückblick auf die Inthronisation des Enmerkara.

Ein solches Ritual zur Bestärkung königlicher Macht wird in mythischer Erzählweise zu einem zentralen Gegenstand des Mythos DIE GÖTTER MACHEN LUGALBANDA ZUM HERRSCHER. Gerade Lugalbanda muss ja als „Toter“60 eine Zeit in einer „Höhle“ im „Gebirge“ (h ur - ru - um kur-ra) zubringen. „Gebirge“ ist im Sumerischen eine übliche Bezeichnung für die Unterwelt, die „Höhle“ steht für das

57 Vgl. dazu den Beitrag von Kärger im vorliegenden Band mit Anhang Nr. 32.

58 Der Ausdruck „Große Erde“ bezeichnet die Unterwelt, die auch als „Sockel“ der Oberwelt verstanden werden kann (so in der Übersetzung von Wilcke 2015, 230).

59 angalta / Innanas Gang zur Unterwelt Z. 1-3, A. Zgoll 2020a.

60 Auf der wörtlichen Erzählebene ist Lugalbanda ein Toter, denn ein Todkranker gilt nach mesopotamischer Sichtweise als Toter, weil er schon von Unterweltsmächten gepackt und in ihren Bann geschlagen ist. Lugalbanda ist tatsächlich von einem Gott geschlagen, befindet sich also schon im Bereich des Todes. Zur übertragenen Sinnebene siehe das Folgende. 
Grab. Dort liegt Lugalbanda wie ein Toter, genau wie der König im Ritual der königlichen Inthronisation und Machterneuerung ${ }^{61}$. Da Lugalbanda kein König ist, geht es bei ihm nicht um königliche Machterneuerung, sondern vielmehr um das Erreichen königlicher Macht. Das Ritual vom Durchgang des Lugalbanda durch die Unterwelt dient ebenfalls, wie das oben geschilderte Ritual von Innanas Erwählung des Lugalbanda zu ihrem Gemahl, der Inthronisation zum Herrscher (vgl. dazu auch unten) .

Hier lässt sich ein Umstand beobachten, der eine ausführlichere Behandlung verdient und hier nur kurz angerissen werden kann. Bei Darstellungen von rituellen Vorgängen in mythischer Form fokussiert die mythische Erzählung in vielen, wenn nicht in den meisten Fällen nicht die Durchführungsebene (die Abfolge der Ritualhandlungen und Ritualworte), sondern die Sinnebene: d. h. das, was diese Handlungen und Worte bedeuten sollen, wofür sie im eigentlichen Sinn stehen. Im vorliegenden Fall: Lugalbanda muss durch den Tod hindurch gehen, um herrscherliche Macht zu erringen. Das Ziel dieses Durchgangs durch den Tod zeigt sich in der Bitte des Toten, als ihm Innana erscheint. Hier geht es nun nicht, wie man erwarten könnte, darum, Innana um Gesundung und Rückkehr ins Leben $\mathrm{zu}$ bitten. Es geht vielmehr um „Haus“ und „Stadt“! Lugalbanda möchte Haus bzw. Stadt „haben“ (Teil 1, Z. 181). Das ist doppeldeutig: Einerseits lässt es sich vordergründig so verstehen, dass er nicht in der Gebirgshöhle sein möchte, sondern in seiner Stadt und bei seinem „Haus“, wo sich seine Verwandten für ihn bei der Göttin verwenden würden. Andererseits wird hier der Wunsch gezeigt, dass Stadt und Tempel mit Lugalbanda verbunden sein sollen, dass sie „seine Stadt“ und „sein Haus“ sein sollen ${ }^{62}$. Das ist das Ziel dieser mythischen Passage und das Ziel des Rituals, auf welches die mythische Hylemsequenz verweist.

Das Ritual des Durchgangs durch das Totenreich dient mithin der jährlichen Erprobung und Bestärkung des Königs und es gehört außerdem zu den königlichen Inthronisationsritualen in Uruk. Gerade in Stellen aus der Enmerkara-Literatur wird deutlich, dass im Kontext der Einführung ins Herrscheramt der Unterweltsgang des Königs mit Innana ein herausragendes Element ist. In der mythischen Darstellung in Enmerkara und Ensuhkešana werden mindestens vier Rituale erwähnt: ein Krönungsritual durch den Gott Enlil, ein Geburtsritual durch den Gott Ninurta und die Geburtsgöttin Aruru, die Heilige Hochzeit von Innana

61 Damit löst sich das Problem, dass Lugalbanda tot und doch nicht tot ist: Auf der Sinnebene des Rituals ist er tot, physisch ist er nicht tot.

62 Auch „Stadt, wo meine Mutter mich geboren hat“ (Teil 1, Z. 182) verweist auf Inthronisationsrituale, die als Geburt durch eine „Mutter“, nämlich die Stadtgöttin, dargestellt werden können, vgl. die Monographie von A. Zgoll zur Religion im antiken Mesopotamien (i. V.). 
und König und der Unterweltsgang beider. Unterweltsgang und Hochzeit auf dem nachtblauen Lager sind hier direkt verbunden ${ }^{63}$. Das Ritual der Heiligen Hochzeit findet genau dort statt, wo sich Innana vom Himmel her kommend in die Unterwelt begeben hat (wörtlich: ,in der Unterwelt aufgestellt hat"), möglicherweise in einem unterirdischen Bau namens Erigal/Irigal ${ }^{64}$ oder Ganzir ${ }^{65}$.

Ritualanweisungen aus dem 1. Jahrtausend, die späte Versionen dieses Rituals sind, belegen, dass der König sich jährlich in einem unterirdisch angelegten Bau aufhalten muss, der als Unterwelt aufzufassen ist ${ }^{66}$. Der Gang in die Unterwelt wird dabei, wie die Zusammenschau weiterer Quellen zeigt, im Rahmen eines inkubierten Traumes angestrebt. Eine solche Praxis ist im Kult der Innana bezeugt, deren Kultangehörige namens kur-ĝara und gala tura während eines inkubierten Traumes lebendig ins Totenreich kommen; gut bezeugt ist es auch im sog. Unterweltstraum des assyrischen Kronprinzen ${ }^{67}$. Im Kontext der hier ausgewerteten mythischen und rituellen Quellen lässt sich erkennen, dass es sich bei diesem Unterweltsgang eines Kronprinzen ebenfalls um ein Inthronisationsritual handelt ${ }^{68}$. Auf die Praxis, mithilfe eines Inkubationsrituals lebendig in die Unterwelt einzutreten, deutet auch die Verbindung aus nächtlichem Lager von König und Innana und ihrem gemeinsamen Unterweltsgang, wie er in Enmerkara und Ensuhkešana angedeutet wird ${ }^{69}$. Aus den Andeutungen der verschiedenen Quellen lässt sich erschließen, dass die rituelle Praxis vorsah, dass der König sich innerhalb eines unterirdischen Bauwerks auf einem kostbaren Lager niederlegte und dass dann eine Inkubation durchgeführt wurde. Während des erhofften Traumes sollte seine Traumseele dann gemeinsam mit der Göttin auf

63 Diese wichtigen Ergebnisse sind den Forschungen von Wilcke 2012, $23-25$ und 78 f., Z. 78-98 zu verdanken.

64 Vgl. dazu auch Wilcke 2012, 78 f. Anm. 146

$65 \mathrm{Zu}$ einem solchen Gebäude in Uruk als Eingang ins Totenreich vgl. A. Zgoll/C. Zgoll 2020, $790 \mathrm{f}$.

66 Vgl. Ambos 2008, 82-85, Ambos 2008a und Wilcke 2012, 25 Anm. 70.

67 Dass diese verschiedenartigen Unterweltsgänge beide im Rahmen von Inkubationsritualen vorgestellt bzw. durchgeführt werden, ist Ergebnis von A. Zgoll 2020b.

68 Daher sieht der Kronprinz dort auch seinen toten Vater oder Vorfahr; der Aufruhr im Land deutet auf Kämpfe um die Thronfolge. In diesem Kontext hat der Text mit dem rituell-mythischen Unterweltsgang legitimierende Funktion.

69 Es liegt auf der Hand, dass es sich bei derartigen Ritualen, wo der König die Unterwelt passieren muss, um Rituale mit Geheimhaltungspflicht handelt, die gerade auch in ihren Details keinesfalls allgemein bekannt waren. 
dem mehrere Doppelstunden dauernden Weg durch die Unterwelt reisen (Enmerkara und Ensuhkešana Z. 87) ${ }^{70}$. Innana war in diesem Kontext in ihrer astralen Erscheinungsform vorzustellen ${ }^{71}$.

Wenn der Abstieg ins Totenreich im Ritual gerade während eines Traumes angestrebt wird, könnte auch im mythisch ausgestalteten Teil des Rituals im Mythos DIE GÖTTER MACHEN LUGALBANDA ZUM HERRSCHER ein solcher Bezug auf Traumgeschehen zu erwarten sein. Und tatsächlich wird ein wesentlicher Teil der Geschehnisse in der Unterwelt als Traumgeschehen geschildert. Lugalbanda führt alleine eine Traum-Inkubation durch ${ }^{72}$. Im Traum erscheint dann der Traumgott und erklärt ihm, wie er den Staatsgöttern und den hohen Gestirngöttern schlachten und opfern muss. Das Geschehen bildet einen Höhepunkt während der Zeit, die Lugalbanda im Totenreich zubringt. Die Erzählebene, betrachtet man sie isoliert, bleibt merkwürdig unverständlich: Wieso sollte ein Inkubationsritual innerhalb der Unterwelt stattfinden, wenn doch die Inkubation dazu dienen soll, in die Unterwelt zu gelangen? Die Merkwürdigkeit im Mythos erklärt sich als Parallele zur Ritualebene: Erst wenn der König im Ritual in ein unterirdisches „Grab“ hinabgestiegen ist, kann die Trauminkubation beginnen. So ist auch hier Lugalbanda schon in der „Höhle“ im „Gebirge“, also im Grab in der Unterwelt, wenn die Inkubation beginnt.

Die Bedeutung dieses Rituals, der Trauminkubation innerhalb des Abstiegs ins Totenreich erschließt sich, wenn man die Schreibungen der Tontafeln genau prüft. Denn gerade hier und nur hier wird, wie Claus Wilcke feststellen konnte, der Protagonist in etlichen Textzeugen nicht als „Mensch“, sondern als „König“ bezeichnet. Das kann kein Zufall sein: „Lugalbanda besitzt bereits königliche Eigenschaften und erwirbt kontinuierlich neue dazu. Er kann darum auch ohne Thron bereits als König gelten“‘3. Anders ausgedrückt: Während der Durchführung des Rituals, des Durchgangs durch das Totenreich, wird Lugalbanda in den Augen verschiedener Schreiber des Textes zum König.

Auch der mythische Vogel Anzu wird Lugalbanda mit einer Macht ausstatten, die ihn zum Herrscher prädestiniert: der Gabe des schnellen Rennens. Spuren verweisen darauf, dass

70 Zur Vorstellung von der menschlichen Traumseele und zu inkubierten Träumen s. ausführlich A. Zgoll 2006, 301-307 (Traumseele) und 308-351 mit 262-276 (Inkubation und Grundlagen). 71 Vgl. Wilcke 2012, 24 und 78.

72 Die Formulierungen zeigen, dass es sich beim Traum des Lugalbanda um einen inkubierten Traum handelt, vgl. A. Zgoll 2006, 100, 113 f., 311-314, 325 mit Anm. 596, 330 (die dort S. 312 angegebene Zeile Lugalbanda-Epos 1:339 entspricht in der neuen Zählung von Wilcke 2015 der Zeile 331).

73 Wilcke 2015, 213. 
auch ein Ritual mit einem Schnell-Lauf zu den herrscherlichen Ritualen gehört: So vollbringt König Šulgi am „Alle-Heiligtümer-Fest“ einen Schnell-Lauf von der Stadt Nippur nach Ur und zurück (Preislied Šulgi $A^{74}$ ), was selbstverständlich nicht als sportliche, sondern als rituelle Glanzleistung angesehen wurde. Dass ein Rennen des Königs als Nachweis königlicher Macht im Ritual dient, findet sich noch bis ins Babylon des 1. Jahrtausends bezeugt: Der König rennt dort beim Neujahrsfest einen Schnell-Lauf. Während dieses Rennens verkörpert er den Hauptgott Marduk, der das die Götter bedrohende Monster Tiāmtu besiegt, was zu Marduks endgültiger Einsetzung als König über die Götter führt. Für das Preislied auf Lugalbanda bedeutet dies, dass auch Lugalbandas Rennen aus der Unterwelt nach Arata und von dort nach Kulaba als mythische Einkleidung bestimmter Inthronisationsrituale aufzufassen ist. Die wesentlichen Abenteuer, die Lugalbanda im Mythos erlebt, haben also neben einer vordergründigen Bedeutung (Reise ins Bergland, Feldzug etc.) eine weitere Sinnebene, die auf Rituale zielt, die Lugalbanda mit königlichen Eigenschaften ausstatten und zum neuen Herrscher machen ${ }^{75}$.

Das Ritual der Heiligen Hochzeit, das Ritual des Unterweltsgangs und das Ritual des Schnell-Laufes sind drei Rituale der Einsetzung eines Menschen durch die Götter zum König; im vorliegenden Erzählstoff werden sie aber als Rituale der Erwählung durch die Götter zum König präsentiert. Dass dies bedeutet, dass der eigentlich noch herrschende König abgesetzt wird, macht die Auswertung des mythischen Erzählstoffes deutlich, auch wenn das Mythenende nicht als Textende präsentiert wird. Es ist der bisherige Herrscher Enmerkara selbst, dem die Ausdeutung der Geschehnisse in den Mund gelegt wird: Innana hat Enmerkara verlassen, als er auf dem Feldzug war ${ }^{76}$. Sie ist nach Kulaba, also in ihre Stadt, zurückgelaufen (Teil 2, 309 f. // 373 f.) ${ }^{77}$. Damit ist klar, dass Enmerkara nicht siegen kann. Aber mehr noch: Innana wird selbst Enmerkaras Waffen zerschmettern

74 Vgl. die Edition von Klein 1981, außerdem Black et al. 2006a.

75 Hierbei gibt es noch eine Beobachtung im Detail anzumerken: Die Fähigkeit, schnell zu rennen, die Lugalbanda aus der Unterwelt entkommen lässt (was diese Fähigkeit mithin als Eigenschaft der Gestirngottheiten erweist, die es schaffen, aus der Unterwelt zu entkommen, vgl. Teil 2, 188 und Woods 2009, 201 Anm. 68), erhält Lugalbanda vom numinosen Anzu-Vogel. Was Anzu ihm hier schenkt, das soll Lugalbanda seinen Freunden und Brüdern nicht sagen, denn: „Dem Guten wohnt Böses inne“ (Teil 2, 216, ÜS Wilcke 2015, 263). Auf der Ebene des Erzählstoffs lässt sich der Grund erkennen: Es ist gut für Lugalbanda, aber böse bzw. schlecht für Enmerkara und für die „Brüder“ Lugalbandas, die anderen Heerführer.

76 Enmerkara wird damit zu einer tragischen Figur, einem tragischen Helden.

77 In dieser Mythenversion wird ein Gegensatz zwischen Uruk und Kulaba aufgebaut: Kulaba wendet sich zurück „,wie Mutterschafe“ vom Krieg gegen Arata (Lugalbanda-Epos Teil 1 Z. 43), im Unterschied zu Uruk, das „wie Auerochsen“ zum Gebirge in den Kampf eilt (Teil 1 Z. 45). Innana kehrt vom Feldzug des Enmerkara nach Kulaba zurück (Teil 1 Z. 197). Kulaba ist „Lugalbandas Stadt“, wohin Utu den Lugalbanda aus dem Totenreich zurückbringen soll (Teil 2 Z. 195). 
(Teil 2, 319 f. // 383 f.). Die Göttin wird also den göttlich eingesetzten Herrscher seines Amtes entheben. Indem sie Lugalbanda als Herrscher eingesetzt hat, ist dieser Machtwechsel schon in Gang gekommen. Denn Innana hat Enmerkara just zu dem Zeitpunkt verlassen, als Lugalbanda in der Höhle im Gebirge war, also während des Inthronisationsrituals für Lugalbanda:

i-gi $i_{4}-i n-z u$ sila gibil? šeg ${ }_{12}^{\text {? }}$ Wahrhaftig: Eine neue Straße! Zum aus $\mathrm{kul}-\mathrm{aba}^{\mathrm{ki}}-\mathrm{še}_{3} \mathrm{na}-\hat{\mathrm{g} e n} \mathrm{n}^{78}$ Ziegeln erbauten ${ }^{79}$ Kulaba ging sie (= Innana) weg (von Enmerkaras Feldzug her) und das hatte wichtige Folgen ${ }^{80}$...

Lugalbanda-Epos Teil 1, Z. 197

Die poetische Formung der Aussage ist ebenso fein ausgestaltet wie die Gesamtanlage des Textes. Für Enmerkara Widriges wird zwar ausgesagt, aber doch möglichst nur angedeutet. Anstatt von der Erwählung des neuen Herrschers zu berichten und davon, dass Innana dem aktuellen Herrscher ihre Gunst und kriegerische Unterstützung im Feldlager entzieht, ist die Rede nur von einer Rückkehr nach Hause, und die „neue Straße“ spielt auf ein neues Ziel, nämlich auf den neuen Herrscher und auf seine Verbindung zu Innana an. Die Art und Weise, wie die Aussage getroffen wird, lässt das Gemeinte ohne Kenntnis des dem Text zugrunde liegenden Erzählstoffs unverständlich bleiben, insofern das Gemeinte eher verschlüsselt als verdeutlicht wird. Ebenso verhält es sich mit der Textstruktur: Auch wenn das Textende in rhetorisch feiner Weise das Mythenende ausspart, so sind doch die im Textverlauf geschickt verteilten und z. T. metaphorisch verschleierten Hyleme eindeutig auf das Ziel des Herrscherwechsels gerichtet.

Dies zeigt sich besonders deutlich, wenn man Textgestaltung und mythischen Inhalt vergleicht. Am Anfang des Textes und des Erzählstoffes findet sich das durative Hylem, dass Enmerkara Herrscher ist. Man vermutet darin, wie häufig in solchen Fällen, ein durativ-konstantes Hylem, das für den gesamten Stoff Geltung hat (was sich in diesem Fall nicht bestätigen wird). Es folgen verschiedene punktuelle Hyleme, die sich als Hyperhyleme zusammenfassen lassen wie folgt: Innana erwählt Lugalbanda als neuen Herrscher und nimmt parallel dazu

In Kulaba (Teil 2 Z. 346) sitzt dann später auch Innana auf ihrem Lager und empfängt Lugalbanda, um ihn zu ihrem Gemahl zu machen.

78 Zitiert nach dem unpublizierten Manuskript der Partitur von Wilcke 2011, vgl. auch Black et al. 2006 Z. 200.

79 Diese Übersetzung des Substantivs „Ziegelwerk“ folgt Wilcke 2015.

80 Zum Verbalpräfix \{na-\} zur Anzeige für den „Effektiv“ vgl. A. Zgoll 2020a, 88 Anm. 18. 
Enmerkara seine Herrschaft weg, während er Krieg gegen Arata führt. Das muss auf Stoffebene zwingend darauf hinauslaufen, dass Innana noch zu Lebzeiten Enmerkaras den Lugalbanda in sein Amt einführt und ihn zum neuen Herrscher anstelle Enmerkaras macht, auch wenn der Text diesen Zielpunkt des Stoffes geflissentlich ausspart. Dass Enmerkara herrscht, erweist sich damit gerade nicht als durativ-konstantes, sondern als durativ-initiales Hylem; es gilt nur am Anfang des Erzählstoffes, wird dann aber durch die nachfolgenden punktuellen Hyleme abgelöst. Am Ende steht als durativ-resultatives Hylem, dass Lugalbanda Herrscher ist. Auch der Feldzug des Enmerkara wird durch Andeutungen am Ende des Textes als Erfolg für Lugalbanda (!) präsentiert:

\section{Stoffchronologie auf Basis ausgewählter repräsentativer Hyleme}

Durativ-initiales Hylem oder „Starthylem“

- Enmerkara ist Herrscher.

\section{Punktuelle Hyperhyleme}

- Enmerkara zieht gegen Arata in den Krieg.

- Innana erwählt Lugalbanda als neuen Herrscher. // Innana nimmt Enmerkara die Herrschaft weg.

- $\quad$ [Innana macht Lugalbanda zum neuen Herrscher]. (implizit ergänzbar)

Durativ-resultatives Hylem oder „Zielhylem“, implizit ergänzbar

- [Lugalbanda ist Herrscher.]

Punktuelles Hyperhylem

- $\quad$ Enmerkaras Truppen besiegen Arata für Lugalbanda ${ }^{81}$.

Durativ-resultatives Hylem oder „Zielhylem“

- $\quad$ Die Beute vom Kriegszug des Enmerkara gehört Lugalbanda ${ }^{82}$.

Die Konsequenz aus diesem Herrscherwechsel findet sich dann in der Unterschrift des Epos eindeutig ausgedrückt. Denn am Ende heißt es „Lugalbanda (sei) Preis!“ Lugalbanda soll demzufolge gepriesen werden, wie sonst Gottheiten und göttliche Herrscher. Der gesamte epische Text mythischen Inhalts bestätigt sich hier als Preislied auf Lugalbanda als (neuen) göttlichen König.

81 Vgl. Teil 2 Z. 401 und Wilcke 2015, 214 und 225.

82 Vgl. Teil 2Z. 406. 
Fazit und Ausblick: Der eigenartige Vergleich Lugalbandas mit einer Gottheit, wenn Lugalbanda zu seinen Brüdern tritt, „wie jemand, der sich aus dem Himmel auf der Erde aufgestellt hat“ (Teil 2 Z. 222), bezeichnet ihn einerseits als numinose Gestalt, macht aber andererseits durch den Vergleich einen Unterschied zum noch amtierenden Herrscher Enmerkara, der im Kontext des Rituals vom Unterweltsgang des Königs als göttliches Wesen präsentiert wird, wenn es direkt und ohne Umschweife heißt, er habe sich vom Himmel in die Große Erde gestellt. Hinzu kommt noch ein weiterer Unterschied: Die Richtung des Sphärenwechsels ist jeweils eine andere. Enmerkara gelangt vom Himmel in die Unterwelt; das hat eine astrale Konnotation, insofern er mit Innana über den Himmel, nämlich den Durchgang am Horizont (vgl. unten) in die Unterwelt eintritt. Auf ritueller Ebene bedeutet das, dass er sich vom Tempel aus - der in Uruk als der „Große Himmel“ angesehen wird ${ }^{83}$ - zum rituellen Unterweltsgang aufmacht. Der Sphärenwechsel zielt hier nach unten.

Anders bei Lugalbanda. Er wird verglichen mit einer Person, die sich vom Himmel auf die Erde stellt, wenn er zu seinen Brüdern zurückkehrt. Doch Lugalbanda war ja gar nicht im oberen Himmel. Die Aussage kann mithin nicht im Sinn eines Himmelssturzes aus dem Zenit gemeint sein. Um die Aussage zu verstehen, muss man sich klar machen, dass der Himmel hier in seiner gesamten Erstrekkung gesehen wird, vom Zenit bis zum Horizont. Am Horizont berühren sich Himmel, Erdoberfläche und Unterwelt, und genau hier ist die Stelle, wo man aus dem kur, der Unterwelt, aufsteigen kann in den Himmel oder auf die Erde treten $\mathrm{kann}^{84}$. Am Horizont treten die Gestirngottheiten wie Innana oder Utu aus der Unterwelt kommend wieder in die Sphäre des Himmels ein. Der Sphärenwechsel, der bei Lugalbanda in den Blick genommen wird, ist also ein zu Enmerkara umgekehrter: In astraler Sichtweise kommt Lugalbanda wie Innana am Horizont, aus der Unterwelt hervor, nur dass er nicht am Himmel aufsteigt, sondern auf die Erd(oberfläche) gelangt, zu seinen Brüdern. Auf ritueller Ebene entspricht dem das Hervorkommen aus einer kultischen Einrichtung, die für die Unterwelt steht, in den Raum des Tempels, den „Großen Himmel“ (vgl. oben), und von dort wieder auf die „Erde“.

Anders als bei Enmerkara liegt der Fokus bei Lugalbanda nicht auf dem Abstieg in die Unterwelt, sondern auf der Rückkehr aus der Unterwelt. Und wie Innana durch ihre Auferstehung aus dem Totenreich das preisende Epitheton $\mathrm{k}_{3}$

83 Vgl. den Beitrag von A. Zgoll, Wie der erste Tempel auf die Erde kommt in diesem Band.

84 Zum Horizont vgl. Woods 2009. 
„strahlend (am Himmel aufgehend), auferstanden“ trägt $^{85}$, so wird gerade dieses Beiwort auch in der weiteren Überlieferung (auch im Lugalbanda-Epos 2,238 und 2,351) zum herausragenden Begleiter des Namens Lugalbanda ${ }^{86}$, der so strahlend wie Innana aus der Unterwelt wieder in den Himmel und von dort auf die Erde zurückgekehrt ist ${ }^{87}$.

Die inhaltliche Fokussierung des vorliegenden Bandes zur Herkunft aus dem Himmel hat sich als Ausgangspunkt für die Entdeckung eines ganzen Geflechts von mythischen Aussagen über Lugalbanda erwiesen, die narrativ plausibilisieren und legitimieren sollen, dass Lugalbanda durch drei Rituale - den Durchgang durch das Totenreich, den Schnell-Lauf und die Heilige Hochzeit - zum rechtmäßigen, von Innana und anderen Göttern erwählten Herrscher wird. Dies

85 Innana/Ištar hat weitere derartige Beiwörter wie z.B. šarrat nipha „Königin des Aufloderns (als wieder sichtbares Gestirn)“, vgl. A. Zgoll 2020 und A. Zgoll/C. Zgoll 2020.

86 Wie bei Innana so wird auch bei Lugalbanda dieses Begleitwort dem Namen vorangestellt eine unübliche Umkehrung der normalen Wortstellung.

87 Passend zur auch sonst dezent-unaufdringlichen Gestaltung des Literaturwerkes wird Lugalbanda nur an ausgewählten Stellen als der „Strahlend-Auferstandene“ bezeichnet. Den Gründen für die Setzung oder Nicht-Setzung des Epithetons $\mathrm{k}_{3} \mathrm{im}_{3}$ Lugalbanda-Epos nachzugehen, ist lohnend. Hier erste Ergebnisse dazu: Die Schreibungen sind in den Textzeugen erstaunlich einheitlich (anders als z.B. die Verwendung oder Auslassung des Gottesdeterminativs). Von den ca. 45 Stellen, wo der Name Lugalbanda genannt wird ( 5 Zusatzzeilen ausgenommen), wird bei etwa einem Drittel das Epitheton „strahlend-auferstanden“ gesetzt. Dazu gehören einschlägige Stellen, wo man einen Bezug auf die Auferstehung des Lugalbanda erwartet: Als er aus der Unterwelt kommend wieder vor seinen Brüdern steht und ihnen Rede und Antwort gibt $(2,238)$ und dort, wo seine Hochzeit mit der „strahlend-auferstandenen“ Innana $(2,347)$ angedeutet wird (2,351 und 353). An manchen Stellen ist die Verwendung des Beiwortes deutlich proleptisch wie auch Lugalbandas Bezeichnung als „König“ (l u gal) schon in 1,331, an der Stelle, wo er sich zum Traumritual niederlegt. Vor allem verwenden die Autoren der Textfassung den Verweis auf Lugalbandas Auferstehung wie einen kommentierenden Zusatz, um Lugalbandas schillernde Persönlichkeit - Schein und wahre Identität - anzudeuten. Die anderen Figuren merken ihm nicht an, dass er aus dem Totenreich zurückgekehrt ist bzw. das Ritual des Unterweltsdurchgangs überstanden hat: Als er zu seiner Truppe zurückkehrt, wird er zunächst ganz unscheinbar, ohne Epitheton beschrieben (2,219.223.225c.227); als aber Lugalbanda den anderen Heerführern antwortet, da blitzt förmlich etwas von dem in ihm liegenden numinosen Status auf ( $\mathrm{mit}_{\mathrm{k}} \mathrm{u}_{3}$, 2,238), auch wenn er innerhalb seiner Antwort - wie ihm der Anzu geraten hatte - seine außergewöhnlichen Erlebnisse verschweigt. Wenn Enmerkara den Lugalbanda aber mit einer Botschaft zu Innana nach Uruk schickt, dann reagieren die anderen Feldherren feindlich; hier ist Lugalbanda mit dem Epitheton $\mathrm{k}_{3}$ auf Erzählebene herausgestellt, was andeutet, dass die anderen potenziellen Rivalen nun etwas von seiner Vorrangstellung ahnen. - Weitere Einblicke in die literarisch herausragende Gestaltung dieses Werkes sind auf der Grundlage der hier unternommenen Neuinterpretation zu erwarten. 
geschieht, während der vorherige Herrscher Enmerkara noch im Amt ist ${ }^{88}$. Das heikle Thema wird in der textlichen Ausgestaltung des Erzählstoffes mit Fingerspitzengefühl behandelt, indem alles, was im Rahmen der Königsideologie Anstoß erregen könnte, übergangen, umgestellt oder nur verschleiert angedeutet wird.

Aus der modernen wissenschaftlichen Perspektive kann man den Erzählstoff vom HEERFÜHRER LUGALBANDA, DER HERRSCHER WIRD, während der bisherige König noch an der Macht ist, als narrative Behandlung des Phänomens der Usurpation beschreiben. Zu möglichen historischen Bezügen sei hier nur angemerkt, dass die Konstellation vom Herrscher Enmerkara und seinem Feldherrn Lugalbanda, der eine neue Dynastie gründet, hervorragend zum Anfang der dritten Dynastie von Ur passt, wo der Feldherr Ur-Namma sich von seinem Herrscher Utu-hegal selbständig macht. Utu-heĝal ist Herrscher über Uruk, Ur-Namma verlegt seine Hauptstadt nach Ur; das würde der Verteilung von Uruk als Stadt des Enmerkara und „Kulaba“ (für Ur) als Stadt des Lugalbanda entsprechen. Zur Verbindung von Ur-Namma mit Lugalbanda passt außerdem, dass Ur-Nammas Sohn Šulgi Lugalbanda (!) als seinen Vater nennt ${ }^{89}$.

Der mesopotamischen Eigenperspektive ist es hingegen gerade darum $\mathrm{zu}$ tun, einen derartigen Tabubruch wie eine Usurpation mit allen Mitteln wenn nicht $\mathrm{zu}$ verschweigen, so doch zumindest abzuschwächen und zu verschleiern - und, mindestens ebenso wichtig, sie göttlich $\mathrm{zu}$ legitimieren und damit $\mathrm{zu}$ rechtfertigen. Während die Analyse der hinter dem Mythos erkennbaren, historisch belegbaren Ritualelemente Lugalbanda als aktiven Handlungsträger erkennen lässt, zeigt der mythische Erzählstoff ihn vornehmlich als Objekt göttlicher Aktionen. Als eigentlicher Handlungsträger wird gerade nicht der menschliche Thronprätendent Lugalbanda gezeigt, sondern die entscheidenden Akteure sind die Göttin Innana, andere Gottheiten wie Utu und Nanna oder der numinose Anzu-Vogel. Innana ist es, die Enmerkaras Königtum beendet, Innana und der Anzu-Vogel sind es, die Lugalbanda durch göttlich-numinose Erwählung (in

88 Darauf kann eventuell auch der Name des Protagonisten anspielen: Lugalbanda bedeutet „junger/zweitrangiger/kleinerer König“, bezeichnet zum vorliegenden Kontext also passend den König in einem Übergangszustand, wo er einerseits schon König wird, andererseits noch nicht vollumfänglich dieses Amt nach außen vertritt.

89 Šulgi A 86a Textzeuge R, entsprechend Šulgi O (Klein 1976, Black et al. 2006b), wonach Šulgi als Bruder von Gilgameš ebenfalls als Sohn des Lugalbanda zu gelten hat. Gösta Gabriel (E-Mail vom 21.10.2020) erwägt, dass das Lugalbanda-Epos, insofern es um den Anfang der Dynastie ringt, einen Nerv der Ur III-Dynastie getroffen hat: „Diese Dynastie fußt auf einer Art ,Ursünde‘, die ggf. durch den Lugalbanda-Mythos ,geheilt‘ werden sollte. Dazu passt auch die allgemeine Fokussierung der Ur III-Könige auf das mythisch-legendarische Uruk.“ 
Form von Heiliger Hochzeit und Unterweltsdurchgang) und durch Machtübertragung (in Form der Fähigkeit, schnell zu rennen wie die Gestirngottheiten) zum neuen Herrscher machen. Die mythische Behandlung der Thematik wird hier funktional eingesetzt, um die Verantwortung für das erzählte Geschehen den Göttern zuzuschreiben, die Lugalbanda zunächst wie eine numinose Persönlichkeit erscheinen lassen, dann eindeutig als numinosen Herrscher einsetzen.

\subsubsection{Beispiel Prometheus: Die Gewinnung des Feuers durch Prometheus bzw. Hermes}

Dass Güter dadurch, dass sie vom Himmel gebracht werden bzw. von Göttern stammen, narrativ als numinos gekennzeichnet werden, eröffnet auch neue Perspektiven für den Mythos vom PROMETHEISCHEN FEUERDIEBSTAHL (aus dem Himmel oder vom Olymp): Das Feuer wird hier als Entität gekennzeichnet, die numinosgöttlichen Charakter trägt. Was folgt daraus für die Interpretation dieses mythischen Stoffes?

In der Regel wird davon ausgegangen, dass dieser Mythos einen entscheidenden Einschnitt in der Menschheitsgeschichte thematisiert, nämlich den Übergang von menschlichen Gemeinschaften, die auf all die verschiedenen Errungenschaften verzichten müssen, die mit dem Feuer und seiner Nutzung in Verbindung stehen, hin zu einer Lebensweise von Menschen, die Feuer zu nutzen in der Lage sind, mit anderen Worten: Dass der Mythos auf die Gabe der Kulturtechnik der Feuerbeherrschung abzielt ${ }^{90}$. Es ist nun die Frage, ob diese Sichtweise nicht zu einseitig ist und zu sehr eine kulturhistorisch-naturwissenschaftliche Betrachtungsweise darstellt, die das Sinnpotenzial des Mythos nicht gänzlich ausschöpft und die emische Perspektive der antiken Menschen zu wenig mit einbezieht. Denn himmlische Gaben sind, wie oben ausgeführt, eben nicht „nur“ etwas Außergewöhnliches, sondern sie sind darüber hinaus etwas Numinoses. Als Kulturtechnik ist das Feuer unbenommen etwas Außergewöhnliches, aber weshalb wird es im Mythos durch seine himmlische bzw. göttliche Abkunft als etwas Numinoses, als etwas Göttliches beschrieben? Warum wird nicht von einem menschlichen Erfinder, sondern von einer göttlichen Gabe gesprochen? Anders gefragt: Was hat das Feuer mit den Göttern zu tun?

90 Vgl. zur Rezeption und Deutungsgeschichte der Prometheus-Gestalt den Überblick mit weiterführenden Hinweisen und Quellenangaben bei H.-K. Lücke/S. Lücke 1999, 680-686, und Reinhardt 2011, 240-242, mit Literaturhinweisen in Anm. 902. 
Zur Annäherung an diese Frage soll ein anderes Beispiel dienen, auf das im vorigen Abschnitt ausführlich eingegangen wurde, nämlich die Herrscher, die in manchen mesopotamischen Mythen beschrieben werden als Personen, die aus dem Himmel kommen oder die Göttern gleichen, die aus dem Himmel kommen. So heißt es vom sumerischen Herrscher Enmerkara, er habe sich „aus dem Himmel in die Große Erde gestellt“, und Lugalbanda wird charakterisiert „wie jemand, der sich aus dem Himmel auf der Erde aufgestellt hat“ (vgl. dazu oben 3.2.1), wodurch angezeigt werden soll, dass diese herausragenden Gestalten numinosen Charakter tragen und als gottgleich zu gelten haben ${ }^{91}$. Diese mesopotamischen Herrschergestalten sind somit nicht nur außergewöhnliche Menschen, sondern sie werden durch solche mythischen Aussagen als numinos, als zugehörig zur göttlichen Sphäre charakterisiert. Damit sind sie wichtige Bindeglieder und Mittler zwischen Göttern und Menschen, wie dies beispielsweise in römischer Tradition in besonderer Weise von König Numa ausgesagt wird ${ }^{92}$.

Analog stellt sich beim Feuer die Frage, worin speziell seine Numinosität begründet liegt. Folgt man dieser Spur, so wird die Gabe des Feuers kaum - zumindest nicht primär und nicht ausschließlich - lediglich etwas vergleichsweise Profanes wie die Etablierung einer neuen Form menschlicher Koch- und Essgewohnheiten oder einer neuen Herstellungstechnik von Essgeschirr anzeigen. Wenn die Gabe des Feuers etwas dezidiert Numinoses darstellt, so ist anzunehmen, dass sie auf Wichtigeres abzielt als nur auf eine neue Zubereitungsmöglichkeit von Speisen oder auf andere profane Zwecke wie das Herstellen von Keramik oder die Metallverarbeitung.

Die Frage ist, in welche Stoßrichtung dann eine solche Betonung der Numinosität des Feuers geht. Die Zusammenschau mit anderen mythischen Erzählstoffen und kulturspezifisches Wissen führen hier weiter: Von Prometheus wird ja nicht nur der Feuerdiebstahl berichtet, sondern ein Mythos berichtet auch davon, dass er den Menschen dabei geholfen hat, dass und auf welche Weise man den Göttern opfern kann. Er ist also auch eine Art „Erschaffer“ des Opferwesens.

Das gestohlene Feuer steht nun genau damit in einem engen Zusammenhang. Denn erst durch die Gabe des Feuers wird es den Menschen möglich, den Göttern auf die richtige Weise Brandopfer darzubringen - auf die Weise nämlich, die aus der tatsächlichen kultischen Praxis der Griechen gut bekannt ist. Wenn Prometheus das Feuer bringt, dann schenkt er also den Menschen damit nicht weniger als eine der wesentlichen Grundlagen für die Interaktion mit den mächtigsten

91 Vgl. dazu den Beitrag von Kärger in Teil 2 dieses Bandes, Abschnitt 4.2.4.1.

92 Vgl. dazu den Beitrag von Jäger in diesem Band (Teil 3), v. a. Abschnitt 6. 
Wesen ihrer Welt. Mit anderen Worten: Es ist nicht in erster Linie eine für menschliche Lebensbelange nützliche Kulturtechnik, die Prometheus den Menschen bringt, sondern vor allem eine der Kommunikation mit den Göttern dienliche Kulttechnik. Das, was die Feuerbeherrschung nicht nur außergewöhnlich, sondern göttlich macht, das ist der Umstand, das sie ein überaus wertvolles Mittel für die Kommunikation mit den Göttern darstellt.

Das Ergebnis lässt sich noch weiter vertiefen. Tatsächlich ist Feuer in den griechisch-römischen Kulturen wichtig, um Rituale richtig ausführen zu können; besonders die Opfer für die Götter. Was man verbrennt, das soll zu den Göttern gelangen; das Feuer ist in diesem Kontext insbesondere auch ein Mittel zum Transport in andere kosmische Räume. Aus dem Kontext von Bestattungen ist diese Funktion des Feuers ebenfalls ersichtlich: Nach dem Tod wird der Tote ebenso wie die Gaben, die man ihm ins Totenreich mitgeben möchte, manchen griechischen Quellen zufolge verbrannt, was offenbar die Funktion hat, dass sich der Übergang in einen anderen kosmischen Bereich, hier in die Unterwelt, vollziehen kann ${ }^{93}$. Das von Prometheus gestohlene Feuer erweist sich im Licht der Ergebnisse zu den Himmelstransfer-Mythen damit aus emischer Perspektive, wie gesagt, nicht nur als wertvolle, aber rein auf den menschlichen Bereich beschränkte Kulturtechnik, sondern aufgrund seiner Numinosität primär als etwas für den Kontakt zu den Göttern Wichtiges.

Dass die Gabe des Feuers in der Sicht griechischer Mythen in einem wesentlichen Konnex nicht mit einer kulinarischen, sondern mit der kultischen Praxis der Verbrennung von Opfertieren steht, zeigt sich auch in der frühesten MythenÜberlieferung über den FeUERdiebstahl DES PROMETHEus bei Hesiod. Sicher ist es kein Zufall, dass bei Hesiod die beiden Mythen von der EINRICHTUNG DES OPFERNS und vom DiEBSTAHL DES FEUERS direkt hintereinander erzählt werden und damit eng aufeinander bezogen sind ${ }^{94}$. Die Rede ist dort von der Opferung eines Rindes und von der Aufteilung der Bestandteile des Tieres auf Anteile, die den Göttern gehören und Anteile, die den Menschen bleiben sollen. Abschließend heißt es ${ }^{95}$ :

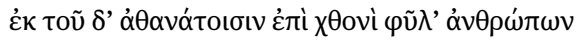

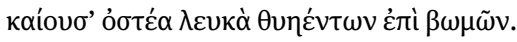

Seitdem aber verbrennen den Unsterblichen auf Erden die Stämme der Menschen weiße Knochen auf opferrauchenden Altären.

93 Vgl. bspw. Hdt. 5,92, ఇ,1-4.

94 Hes. theog. 521-569.

95 Hes. theog. $556 \mathrm{f}$. 
Dieses „seitdem“ kann aber nicht sofort, sondern erst zu einem späteren Zeitpunkt eintreten, denn Zeus, der sich bei der Aufteilung des Rindes von Prometheus hintergangen fühlt ${ }^{96}$, enthält den Menschen das Feuer vor, so dass es zunächst eben gerade nicht zu einer solchen Opferverbrennung durch die Menschen kommen kann. Direkt im Anschluss wird dann vom Diebstahl des Feuers durch Prometheus berichtet, so dass erst ab dann, also erst nach dem Feuerdiebstahl, oder, zugespitzt formuliert: erst aufgrund des Feuerdiebstahls durch Prometheus die Praxis der Verbrennung des vorher eingerichteten Tieropfers von den Menschen überhaupt durchgeführt werden kann.

Eine weitere Bestätigung erhält dieses Ergebnis, dass die Gabe des Feuers in erster Linie die für die Interaktion mit den Göttern wichtige Brandopferung ermöglichen soll, außerdem noch durch einen Vergleich mit einem anderen Mythos. In der griechischen Mythologie gibt es nämlich einen weiteren MYTHOS zUR GEWINNUNG DES FEUERS, der im Homerischen Hermes-Hymnos überliefert wird: Hier ist nicht Prometheus der Protagonist, sondern der Gott Hermes. Hermes muss das Feuer auch nicht aus dem Herrschaftsbereich des Zeus stehlen, sondern als noch kleines Kind erfindet er selbst die Technik des Feuermachens, und schließlich bringt Hermes das Feuer nicht den Menschen, sondern er benutzt es, zumindest auf den ersten Blick betrachtet, zunächst einmal für sich selbst ${ }^{97}$. Bei all der Verschiedenheit aber gibt es eine auffällige Gemeinsamkeit: Hermes erfindet das Feuer, um das Fleisch von zwei Kühen zu braten - aber nicht, um dieses Fleisch dann selbst zu essen, sondern fast im Gegenteil: Obwohl es ihn sehr gelüstet, von dem köstlich duftenden Fleisch zu essen, rührt er nichts davon an, sondern bringt das Fleisch, aufgeteilt in zwölf Portionen, als Opfer dar - gemessen an den zwölf Opfer-Portionen soll dies ganz offensichtlich als Opfer für die zwölf olympischen Götter zu verstehen sein.

Damit steht auch in dieser Mythenvariante das Feuer und seine Erfindung in einem direkten Zusammenhang nicht mit der Kochkunst, sondern mit der Götterverehrung, entsprechend dem Aufwand des Opfers hier im Homerischen HermesHymnos wie in Hesiods Theogonie speziell mit der Verehrung der obersten Götter. Und damit ist das Feuer aus Sicht dieser mythischen Stoffe nicht so sehr als Kulturgut, sondern eher als ein „Kultgut“ anzusehen.

96 Zum sog. „Opferbetrug“ des Prometheus bei Hesiod s. ausführlich C. Zgoll 2019, 340-350.

97 Hom. h. 4,108-137. 


\subsubsection{Mythen über eine Herkunft aus dem Himmel erzählen und plausibilisieren Numinosität}

Mythen über eine Herkunft aus dem Himmel (bzw. von den Göttern) bezeichnen die Numinosität derjenigen Entitäten - Personen, Dinge, Einrichtungen -, die vom Himmel kommen, wie die vorangehenden Abschnitte allgemein und exemplarisch deutlich gemacht haben: Was vom Himmel oder von den Göttern kommt, ist durch diese mythische Erzählweise als numinos bzw. (im Fall von göttlichen Personen) als göttlich anzusehen.

Mythische Erzählstoffe (in verschiedenen medialen Konkretionen) deklarieren dabei nicht Numinosität oder Göttlichkeit, wie dies bspw. ein theologischer Traktat tun würde („X ist göttlich“), sie preisen diese auch nicht in Form isolierter Prädikationen, wie dies etwa in bestimmten religiösen Hymnen der Fall wäre ("Du bist Gott"), sondern sie erzählen davon ${ }^{98}$. Numinosität zu erzählen bedeutet, dass diese Mythen den numinosen Charakter einer Person, einer Sache oder eines Phänomens narrativ plausibel machen. Der in antiken Mythen vorkommende Vorgang „X kommt vom Himmel (zu/nach Y)“ ist nicht die einzige, aber eine wichtige mythische Einkleidung und Plausibilisierung für Numinosität.

\section{Literaturverzeichnis}

Ambos, C., 2008, Der König im Gefängnis und das Neujahrsfest im Herst. Mechanismen der Legitimation des babylonischen Herrschers im 1. Jahrtausend v. Chr. und ihre Geschichte, Dresden.

Ambos, C., 2008a, Das „Neujahrs“-Fest zur Jahresmitte und die Investitur des Königs im Gefängnis, in: Prechel, D. (Hg.), Fest und Eid: Instrumente der Herrschaftssicherung im Alten Orient. Kulturelle und Sprachliche Kontakte 3, Würzburg, 1-12.

Attinger, P. 2014, Iddin-Dagan A, in: N. Koslova, E. Vizirova, and G. Zólyomi (Hg.), Babel und Bibel 8: Studies in Sumerian Language and Literature. Festschrift Joachim Krecher, 11-82. (https://zenodo.org/record/3593446\#.X94eABYxIPY)

Benjamin, W., 1965, Zur Kritik der Gewalt, in: Benjamin, W., Zur Kritik der Gewalt und andere Aufsätze, mit einem Nachw. versehen von Herbert Marcuse, Edition Suhrkamp 103, Frankfurt am Main, 78-94 (Ndr. d. Erstpubl. 1921 in Archiv für Sozialwissenschaft und Sozialpolitik 47/3, 809-832).

98 Zur Grundfunktion von Mythen, Erfahrungsgegenstände in Form von Erzählungen zum Ausdruck zu bringen und kommunizierbar zu machen, s. C. Zgoll 2019, 420-422; zu verschiedenen Arten der Transformation von Erfahrungsgegenständen durch die narrative Einkleidung s. ebd., 391-394. Konkrete Beispiele für die narrative Exemplifikations-Funktion von Mythen bieten Gabriel 2018 (für Mesopotamien) und zahlreiche Einzeluntersuchungen im vorliegenden Band. 
Beth, K., 1935, Mythologie und Mythus, Handwörterbuch des deutschen Aberglaubens 6, 720752.

Binsbergen, W. van/Wiggermann F. A. M., 1999, Magic in History. A Theoretical Perspective, and Its Application to Ancient Mesopotamia, in: Abusch, T./van der Toorn, K. (Hg.), Mesopotamian Magic. Textual, Historical, and Interpretative Perspectives, Ancient Magic and Divination 1, Groningen, 3-34.

Black, J., et al. 2006, ETCSL 1.8.2.1, Lugalbanda in the mountain cave [abgerufen: 15.12.2020]. Black, J., et al. 2006a, ETCSL 2.4.2.01, A praise poem of Šulgi (Šulgi A) [abgerufen: 15.12.2020]. Black, J., et al. 2006b, ETCSL 2.4.2.15, A praise poem of Šulgi (Šulgi 0) [abgerufen: 15.12.2020]. Black, J., et al. 2006c, ETCSL 2.5.3.1, A šir-namursaĝa to Ninsiana for Iddin-Dagan (Iddin-Dagan A) [abgerufen: 15.12.2020].

Blumenberg, H., 1979, Arbeit am Mythos, Frankfurt am Main (Ndr. d. 5. Aufl. 1990: 1996).

Bottéro, J., 2001, Religion in Ancient Mesopotamia, Chicago (franz. Orig.: La plus vieille religion: En Mésopotamie, Paris 1998).

Gabriel, G. I., 2018, An Exemplificational Critique of Violence. Re-reading the Old Babylonian Epic Inūma ilū awīlum (a.k.a. Epic of Atramhasīs), in: Gabriel, G. I. (Hg.), Approaching a Critique of Mesopotamian Reason. Proceedings of the Volkswagen Fellowship Symposium, held at Harvard University, April 20-21, 2017, in: Journal of Ancient Near Eastern History 5 (1-2), 179-213.

Hawthorn, A., 2020, The Fish and the Tamarisk: Sexual and Celestial Symbolism in "Lugalbanda and the Anzu Bird", in: Naether, F. (Hg.) Cult Practices in Ancient Literatures: Egyptian, Near Eastern and Graeco-Roman Narratives in a Cross-Cultural Perspective. Proceedings of a Workshop at the Institute for the Study of the Ancient World, New York, May 1617, 2016, ISAW Papers 18.

Johannsen, D., 2008, Das Numinose als kulturwissenschaftliche Kategorie. Norwegische Sagenwelt in religionswissenschaftlicher Deutung, Religionswissenschaft heute 6, Stuttgart.

Klein, J. 1976, Šulgi and Gilgameš: Two Brother-Peers (Šulgi 0), in: B.L. Eichler (Hg.), Kramer Anniversary Volume, AOAT 25, 271-292.

Klein, J. 1981, Three Šulgi Hymns. Sumerian Royal Hymns Gloryfying King Šlgi of Ur, Bar-llan.

Lücke, H.-K./Lücke, S., 1999, Antike Mythologie: Ein Handbuch. Der Mythos und seine Überlieferung in Literatur und bildender Kunst, Hamburg.

Mlttermayer, C., 2009, Enmerkara und der Herr von Arata. Ein ungleicher Wettstreit, Orbis biblicus et orientalis 239, Fribourg/Göttingen.

Otto, R., 1917, Das Heilige. Über das Irrationale in der Idee des Göttlichen und sein Verhältnis zum Rationalen, Breslau.

Radt, S., 1985, Tragicorum Graecorum Fragmenta (TrGF), vol. 3: Aeschylus, Göttingen.

Reinhardt, U., 2011, Der antike Mythos. Ein systematisches Handbuch, Paradeigmata 14, Freiburg.

Wiggermann siehe van Binsbergen.

Wilcke, C., 1969, Das Lugalbandaepos, Wiesbaden.

Wilcke, C., 2011, Lugalbanda-Partitur (unpubliziertes Manuskript).

Wilcke, C., 2012, The Sumerian Poem Enmerkar and En-suhkeš-ana: Epic, Play, Or? Stage Craft at the Turn from the Third to the Second Millennium B.C. (= AOS Essay 12), New Haven.

Wilcke, C., 2015, Vom klugen Lugalbanda, in: K. Volk, Erzählungen aus dem Land Sumer, Wiesbaden, 203-272. 
Woods, C., 2009, At the Edge of the World: Cosmological Conceptions of the Eastern Horizon in Mesopotamia, in: JANER 9, 183-239.

Zgoll, A., 2006, Traum und Welterleben im antiken Mesopotamien. Traumtheorie und Traumpraxis im 3. - 1. Jt. v. Chr. als Horizont einer Kulturgeschichte des Träumens, in: Alter Orient und Altes Testament 333, Münster.

Zgoll, A., 2012, Welt, Götter und Menschen in den Schöpfungsentwürfen des antiken Mesopotamien, in: Schmid, K. (Hg.), Schöpfung, Themen der Theologie 4, Tübingen, 17-70.

Zgoll, A., 2013, Fundamente des Lebens. Vom Potential altorientalischer Mythen, in: Zgoll, A./ Kratz, R. G. (Hg.), Arbeit am Mythos. Leistung und Grenze des Mythos in Antike und Gegenwart, unter Mitarbeit von K. Maiwald, Tübingen, 79-107.

Zgoll, A., 2020, Condensation of Myths. A Hermeneutic Key to a Myth about Innana and the Instruments of Power (me), Incorporated in the Epic angalta, in: Sommerfeld, W. (Hg.), Dealing with Antiquity - Past, Present, and Future, Proceedings der 63. Rencontre Assyriologique Internationale Marburg 2017, Alter Orient und Altes Testament 460, Münster, 427447.

Zgoll, A., 2020a, Durch Tod zur Macht, selbst über den Tod. Mythische Strata von Unterweltsgang und Auferstehung der Innana/Ištar in sumerischen und akkadischen Quellen, in: Zgoll, A./Zgoll, C. (Hg.), Mythische Sphärenwechsel. Methodisch neue Zugänge zu antiken Mythen in Orient und Okzident, Mythological Studies 2, Boston/Berlin, 83-159.

Zgoll, A., 2020b, Wege der Lebenden ins „Land ohne Wiederkehr“ in Mythen und Ritualen der mesopotamischen Antike: Der Unterweltstraum eines assyrischen Kronprinzen und Innana(k)s Gang zur Unterwelt, in: M. Egeler/W. Heizmann (Hg.), Between the Worlds. Contexts, Sources, and Analogues of Scandinavian Otherworld Journeys. Ergänzungsbände zum Reallexikon der Germanischen Altertumskunde, Berlin/Boston, 432-457.

Zgoll, A./Zgoll, C., 2020, Innana-Ištars Durchgang durch das Totenreich in Dichtung und Kult. Durch Hylemanalysen zur Erschließung von Spuren mythischer Stoffvarianten in kultischer Praxis und epischer Verdichtung, in: Arkhipov, I./Kogan, L./Koslova, N. (Hg.), The Third Millennium: Studies in Early Mesopotamia and Syria in Honor of Manfred Krebernik and Walter Sommerfeld, Cuneiform Monographs 50, Leiden, 749-801.

Zgoll, C., 2019, Tractatus mythologicus. Theorie und Methodik zur Erforschung von Mythen als Grundlegung einer allgemeinen, transmedialen und komparatistischen Stoffwissenschaft, Mythological Studies 1 (https://doi.org/10.1515/9783110541588), Berlin/Boston. 
